\title{
Discrete unified gas kinetic scheme for all Knudsen number flows. II. Thermal compressible case
}

\author{
Zhaoli Guo, ${ }^{1,2, *}$ Ruijie Wang, ${ }^{3, \dagger}$ and Kun $\mathrm{Xu}^{2, \ddagger}$ \\ ${ }^{1}$ State Key Laboratory of Coal Combustion, Huazhong University of Science and Technology, Wuhan 430074, China \\ ${ }^{2}$ Department of Mathematics, Hong Kong University of Science and Technology, Clear Water Bay, Hong Kong, China \\ ${ }^{3}$ Nano Science and Technology Program, Hong Kong University of Science and Technology, Clear Water Bay, Hong Kong, China
}

(Received 22 June 2014; revised manuscript received 1 December 2014; published 31 March 2015)

\begin{abstract}
This paper is a continuation of our work on the development of multiscale numerical scheme from low-speed isothermal flow to compressible flows at high Mach numbers. In our earlier work [Z. L. Guo et al., Phys. Rev. E 88, 033305 (2013)], a discrete unified gas kinetic scheme (DUGKS) was developed for low-speed flows in which the Mach number is small so that the flow is nearly incompressible. In the current work, we extend the scheme to compressible flows with the inclusion of thermal effect and shock discontinuity based on the gas kinetic Shakhov model. This method is an explicit finite-volume scheme with the coupling of particle transport and collision in the flux evaluation at a cell interface. As a result, the time step of the method is not limited by the particle collision time. With the variation of the ratio between the time step and particle collision time, the scheme is an asymptotic preserving (AP) method, where both the Chapman-Enskog expansion for the Navier-Stokes solution in the continuum regime and the free transport mechanism in the rarefied limit can be precisely recovered with a second-order accuracy in both space and time. The DUGKS is an idealized multiscale method for all Knudsen number flow simulations. A number of numerical tests, including the shock structure problem, the Sod tube problem in a whole range of degree of rarefaction, and the two-dimensional Riemann problem in both continuum and rarefied regimes, are performed to validate the scheme. Comparisons with the results of direct simulation Monte Carlo (DSMC) and other benchmark data demonstrate that the DUGKS is a reliable and efficient method for multiscale flow problems.
\end{abstract}

DOI: 10.1103/PhysRevE.91.033313

PACS number(s): 47.11.St, 47.45.-n, 47.61.-k

\section{INTRODUCTION}

It is a challenging problem to model and simulate nonequilibrium flows over a wide range of Knudsen number regimes. The difficulty arises from the different temporal and spatial scales associated with different flow physics. For instance, in the transition and free-molecule limit the kinetic physics in the particle mean-free-path scale has to be considered, and in the continuum flow regime the hydrodynamic mechanism in a macroscopic dissipative length scale needs to be modeled. For a multiscale flow study, one popular numerical strategy is to use a hybrid approach, where the computational domain is divided into subdomains with different governing equations [1]. For instance, in hybrid particle-continuum approaches, the domain is divided into some macro and micro subdomains, where particle-based methods, such as molecular dynamics (MD) or direct simulation Monte Carlo (DSMC), are used in the micro subdomain, and the continuum Navier-Stokes equations are used in the macro subdomain. Usually a buffer zone is employed in the hybrid method between different subdomains with predefined flow information exchange strategies [2-6]. A common feature of the hybrid methods is that they are based on numerical coupling of solutions from different flow regimes, which are limited to systems with a clear scale separation. This strategy may encounter great difficulties for flows with a continuous variation of flow physics [1].

Recently, some efforts have been made to develop numerical schemes for multiscale flows based on kinetic models

\footnotetext{
*zlguo@mail.hust.edu.cn

${ }^{\dagger}$ ruijie.wang@ust.hk

${ }^{\ddagger}$ makxu@ust.hk
}

(e.g., the Boltzmann equation or simplified models). These kinetic schemes attempt to provide a unified flow description in different regimes through the discretization of the same kinetic equation in order to avoid the difficulties of hybrid methods. One of the kinetic schemes is the discrete ordinate method (DOM) [7-9], which presents accurate solutions in the kinetic regime, but may have difficulties for near continuum flow computation due to the use of cell size and time step on the order of particle mean free path and collision time. In order to overcome this problem, many asymptotic preserving (AP) schemes have been developed (e.g., [10-13]), which mostly can recover the Euler solutions in the continuum limit but may encounter difficulties for recovering the Navier-Stokes solutions. Therefore it is still desirable to design kinetic schemes that can work efficiently and accurately in a wide range of flow regimes.

The unified gas kinetic scheme (UGKS) is a dynamical multiscale method with the inclusion of flow physics from both continuum and free-molecular regimes in its algorithm development [14,15]. The UGKS is a finite-volume scheme for the Boltzmann model equations [16], and the particle velocity space is discretized into a discrete velocity set, like the DOM. However, in the update of the discrete distribution function the flow physics from the coupled particle transport and collision process is followed, and with the variation of scales the numerical time step is not limited by the particle collision term. Furthermore, the UGKS adopts the local integral solution of the kinetic model equation in the reconstruction of the time-dependent gas distribution function at a cell interface for flux evaluation, and this flux function includes the flow physics from the kinetic particle free transport to hydrodynamic wave propagation. In the original UGKS, besides the update of gas distribution function, the corresponding macroscopic variables 
are updated as well, which requires additional computation cost in comparison with DOM method.

An alternative, simpler UGKS method, i.e., the so-called discrete unified gas kinetic scheme (DUGKS), has been proposed recently [17]. This scheme is also a finite-volume discretization of the Boltzmann-Bhatnagar-Gross-Krook (BGK) equation. But different from the UGKS [14], the flow update is based on the evolution of a modified distribution function instead of the original one, which removes the implicit treatment of the collision term inside each control volume in the UGKS. At the same time, the evolution of macroscopic variables is not required in DUGKS. Furthermore, the distribution function at a cell interface in DUGKS is constructed from the averaging along the characteristic line, instead of the local integral solution in UGKS. As a result, the formulation can be much simplified without scarifying the multiscale dynamics. The DUGKS has the same modeling mechanism as the original UGKS. The DUGKS has been applied successfully to a number of gas flows, ranging from continuum to transition regimes [17].

The previous DUGKS is designed for low-speed isothermal flow where the Mach number is sufficiently small so that the flow is nearly incompressible and temperature variations can be neglected. However, in nonequilibrium flows, temperature change may be important in both aerospace and microflow applications. Under such a circumstance, it is necessary to capture the thermal effect in addition to the fluid dynamics. In this work, a full DUGKS is developed for nonequilibrium gas flows with temperature variation. The scheme is constructed based on the BGK-Shakhov model, which can yield a correct Prandtl number in the continuum regime [18]. The rest of this paper is organized as follows. In Sec. II, the full DUGKS is presented with the analysis of the properties of the scheme. In Sec. III, a number of numerical tests, ranging from subsonic to hypersonic flows in different Knudsen regimes, are conducted to validate the method. In Sec. IV, a brief summary is given.

\section{BGK-SHAKHOV MODEL}

In kinetic theory, the BGK model [16] uses only one single relaxation time, which leads to a fixed unit Prandtl number. In order to overcome this limitation, a number of improved models, such as the BGK-Shakhov model [18] and the ellipsoidal statistical model [19], have been proposed based on different physical considerations. In $D$-dimensional space, the BGK-Shakhov model can be expressed as

$$
\frac{\partial f}{\partial t}+\xi \cdot \nabla f=\Omega \equiv-\frac{1}{\tau}\left[f-f^{S}\right],
$$

where $f=f(\boldsymbol{x}, \boldsymbol{\xi}, \boldsymbol{\eta}, \boldsymbol{\zeta}, t)$ is the velocity distribution function for particles moving in $D$-dimensional physical space with velocity $\boldsymbol{\xi}=\left(\xi_{1}, \ldots, \xi_{D}\right)$ at position $\boldsymbol{x}=\left(x_{1}, \ldots, x_{D}\right)$ and time $t$. Here $\boldsymbol{\eta}=\left(\xi_{D+1}, \ldots, \xi_{3}\right)$ is a vector of length $L=$ $3-D$, consisting of the rest components of the particle velocity $\left(\xi_{1}, \xi_{2}, \xi_{3}\right)$ in three-dimensional (3D) space; $\zeta$ is a vector of length $K$ representing the internal degree of freedom of molecules; $\tau$ is the relaxation time relating to the dynamic viscosity $\mu$ and pressure $p$ with $\tau=\mu / p$, and $f^{S}$ is the
Shakhov equilibrium distribution function given by

$$
\begin{aligned}
f^{S} & =f^{e q}\left[1+(1-\operatorname{Pr}) \frac{\boldsymbol{c} \cdot \boldsymbol{q}}{5 p R T}\left(\frac{c^{2}+\eta^{2}}{R T}-5\right)\right] \\
& =f^{e q}+f_{\mathrm{Pr}},
\end{aligned}
$$

where $f^{e q}$ is the Maxwellian distribution function, $\operatorname{Pr}$ is the Prandtl number, $\boldsymbol{c}=\boldsymbol{\xi}-\boldsymbol{u}$ is the peculiar velocity with $\boldsymbol{u}$ being the macroscopic flow velocity, $\boldsymbol{q}$ is the heat flux, $R$ is the gas constant, and $T$ is the temperature. The Maxwellian distribution function $f^{e q}$ is given by

$$
f^{e q}=\frac{\rho}{(2 \pi R T)^{(3+K) / 2}} \exp \left(-\frac{c^{2}+\eta^{2}+\zeta^{2}}{2 R T}\right),
$$

where $\rho$ is the density. The conservative flow variables are defined by the moments of the distribution function,

$$
\boldsymbol{W}=\left(\begin{array}{c}
\rho \\
\rho \boldsymbol{u} \\
\rho E
\end{array}\right)=\int \boldsymbol{\psi}(\boldsymbol{\xi}, \boldsymbol{\eta}, \zeta) f d \boldsymbol{\xi} d \boldsymbol{\eta} d \zeta
$$

where $\boldsymbol{\psi}=\left(1, \boldsymbol{\xi},\left(\xi^{2}+\eta^{2}+\zeta^{2}\right) / 2\right)^{T}$ is the collision invariant, $\rho E=\rho u^{2} / 2+\rho \epsilon$ is the total energy, and $\epsilon=c_{V} T$ is the international energy, with $c_{V}$ being the specific heat capacity at constant volume. The pressure is related to density and temperature through an ideal equation of state, $p=\rho R T$, and the heat flux is defined by

$$
\boldsymbol{q}=\frac{1}{2} \int \boldsymbol{c}\left(c^{2}+\eta^{2}+\zeta^{2}\right) f d \boldsymbol{\xi} d \eta d \zeta
$$

The specific heat capacities at constant pressure and volume are $c_{p}=(5+K) R / 2$ and $c_{V}=(3+K) R / 2$, respectively, and so the specific heat ratio is

$$
\gamma=\frac{c_{p}}{c_{V}}=\frac{K+5}{K+3} .
$$

The stress tensor $\boldsymbol{\tau}$ is defined from the second-order moment of the distribution function,

$$
\boldsymbol{\tau}=\int \boldsymbol{c c}\left(f-f^{e q}\right) d \boldsymbol{\xi} d \boldsymbol{\eta} d \zeta .
$$

The dynamic viscosity $\mu$ usually depends on the intermolecular interactions. For example, for hard-sphere (HS) or variable hard-sphere (VHS) molecules,

$$
\mu=\mu_{\text {ref }}\left(\frac{T}{T_{\text {ref }}}\right)^{\omega}
$$

where $\omega$ is the index related to the HS or VHS model, $\mu_{\text {ref }}$ is the viscosity at the reference temperature $T_{\text {ref }}$.

The evolution of the distribution function $f$ depends only on the $D$-dimensional particle velocity $\boldsymbol{\xi}$ and is irrelevant to $\boldsymbol{\eta}$ and $\zeta$. In order to remove the dependence of the passive variables, two reduced distribution functions can be introduced [7],

$$
\begin{aligned}
& g(\boldsymbol{x}, \boldsymbol{\xi}, t)=\int f(\boldsymbol{x}, \boldsymbol{\xi}, \boldsymbol{\eta}, \zeta, t) d \boldsymbol{\eta} d \boldsymbol{\zeta} \\
& h(\boldsymbol{x}, \boldsymbol{\xi}, t)=\int\left(\eta^{2}+\zeta^{2}\right) f(\boldsymbol{x}, \boldsymbol{\xi}, \boldsymbol{\eta}, \boldsymbol{\zeta}, t) d \eta d \boldsymbol{\zeta}
\end{aligned}
$$


From Eq. (4), we can obtain that

$$
\begin{aligned}
\rho & =\int g d \boldsymbol{\xi}, \quad \rho \boldsymbol{u}=\int \boldsymbol{\xi} g d \boldsymbol{\xi}, \\
\rho E & =\frac{1}{2} \int\left(\xi^{2} g+h\right) d \xi,
\end{aligned}
$$

and the heat flux $\boldsymbol{q}$ and the stress tensor can be computed as

$$
\boldsymbol{q}=\frac{1}{2} \int \boldsymbol{c}\left(c^{2} g+h\right) d \boldsymbol{\xi}, \quad \boldsymbol{\tau}=\int \boldsymbol{c} \boldsymbol{c}\left(g-g^{e q}\right) d \boldsymbol{\xi},
$$

where $g^{e q}$ is the reduced equilibrium distribution function given below.

The evolution equations for $g$ and $h$ can be obtained from Eq. (1),

$$
\begin{aligned}
& \frac{\partial g}{\partial t}+\xi \cdot \nabla g=\Omega_{g} \equiv-\frac{1}{\tau}\left[g-g^{S}\right], \\
& \frac{\partial h}{\partial t}+\xi \cdot \nabla h=\Omega_{h} \equiv-\frac{1}{\tau}\left[h-h^{S}\right],
\end{aligned}
$$

where the reduced Shakhov distribution functions $g^{S}$ and $h^{S}$ are given by

$$
\begin{aligned}
g^{S}(\boldsymbol{x}, \boldsymbol{\xi}, t) & =\int f^{S}(\boldsymbol{x}, \boldsymbol{\xi}, \boldsymbol{\eta}, \boldsymbol{\zeta}, t) d \boldsymbol{\eta} d \boldsymbol{\zeta}=g^{e q}+g_{\mathrm{Pr}} \\
h^{S}(\boldsymbol{x}, \boldsymbol{\xi}, t) & =\int\left(\eta^{2}+\zeta^{2}\right) f^{S}(\boldsymbol{x}, \boldsymbol{\xi}, \boldsymbol{\eta}, \zeta, t) d \eta d \zeta \\
& =h^{e q}+h_{\mathrm{Pr}}
\end{aligned}
$$

with

$$
\begin{aligned}
g^{e q} & =\int f^{e q} d \boldsymbol{\eta} d \boldsymbol{\rho} \\
& =\frac{\rho}{(2 \pi R T)^{D / 2}} \exp \left[-\frac{(\boldsymbol{\xi}-\boldsymbol{u})^{2}}{2 R T}\right] \\
h^{e q} & =\int\left(\eta^{2}+\zeta^{2}\right) f^{e q} d \eta d \zeta=(K+3-D) R T g^{e q}, \\
g_{\operatorname{Pr}} & =\int f_{\operatorname{Pr}} d \boldsymbol{\eta} d \zeta \\
& =(1-\operatorname{Pr}) \frac{\boldsymbol{c} \cdot \boldsymbol{q}}{5 p R T}\left[\frac{c^{2}}{R T}-D-2\right] g^{e q},
\end{aligned}
$$

and

$$
\begin{aligned}
h_{\mathrm{Pr}}= & \int\left(\eta^{2}+\zeta^{2}\right) f_{\mathrm{Pr}} d \eta d \zeta \\
= & (1-\operatorname{Pr}) \frac{\boldsymbol{c} \cdot \boldsymbol{q}}{5 p R T}\left[\left(\frac{c^{2}}{R T}-D\right)(K+3-D)-2 K\right] \\
& \times R T g^{e q} .
\end{aligned}
$$

With the definitions of the conserved variables, it is easy to verify that the collision terms $\Omega_{g}$ and $\Omega_{h}$ satisfy the following conservative laws:

$$
\begin{aligned}
\int \Omega_{g} d \xi & =0, \quad \int \xi \Omega_{g} d \xi=\mathbf{0}, \\
\int\left(\xi^{2} \Omega_{g}+\Omega_{h}\right) d \xi & =0 .
\end{aligned}
$$

\section{DISCRETE UNIFIED GAS KINETIC SCHEME}

\section{A. Updating of the cell-averaged distribution function}

The full DUGKS is constructed based on the two reduced kinetic equations (13). The scheme is a finite-volume formulation of the kinetic equations. For simplicity, we rewrite Eq. (12) in the following form:

$$
\frac{\partial \phi}{\partial t}+\xi \cdot \nabla \phi=\Omega \equiv-\frac{1}{\tau}\left[\phi-\phi^{S}\right],
$$

for $\phi=g$ or $h$. The domain is decomposed into a set of control volumes (cells), then the integration of Eq. (16) over cell $j$ centering at $\boldsymbol{x}_{j}$ from time $t_{n}$ to $t_{n+1}$ with time step $\Delta t$ leads to

$$
\begin{gathered}
\phi_{j}^{n+1}(\boldsymbol{\xi})-\phi_{j}^{n}(\boldsymbol{\xi})+\frac{\Delta t}{\left|V_{j}\right|} \boldsymbol{F}^{n+1 / 2}(\boldsymbol{\xi}) \\
=\frac{\Delta t}{2}\left[\Omega_{j}^{n+1}(\boldsymbol{\xi})+\Omega_{j}^{n}(\boldsymbol{\xi})\right],
\end{gathered}
$$

where the midpoint rule is used for the time integration of the convection term and the trapezoidal rule for the collision term. Such treatment ensures the scheme is of second-order accuracy in time. Here

$$
\boldsymbol{F}^{n+1 / 2}(\boldsymbol{\xi})=\int_{\partial V_{j}}(\boldsymbol{\xi} \cdot \boldsymbol{n}) \phi\left(\boldsymbol{x}, \boldsymbol{\xi}, t_{n+1 / 2}\right) d \boldsymbol{S}
$$

is the microflux across the cell interface, where $\left|V_{j}\right|$ and $\partial V_{j}$ are the volume and surface of cell $V_{j}, \boldsymbol{n}$ is the outward unit vector normal to the surface, and $\phi_{j}$ and $\Omega_{j}$ are the cellaveraged values of the distribution function and collision term, respectively, e.g.,

$$
\phi_{j}^{n}(\boldsymbol{\xi})=\frac{1}{\left|V_{j}\right|} \int_{V_{j}} \phi\left(\boldsymbol{x}, \boldsymbol{\xi}, t_{n}\right) d \boldsymbol{x} .
$$

The update rule given by Eq. (17) is implicit due to the term $\Omega_{j}^{n+1}$, which requires the conserved variables $\boldsymbol{W}_{j}^{n+1}$. In order to remove this implicity, we employ a technique as used in the development of the isothermal DUGKS [17], i.e., we introduce a distribution function,

$$
\tilde{\phi}=\phi-\frac{\Delta t}{2} \Omega=\frac{2 \tau+\Delta t}{2 \tau} \phi-\frac{\Delta t}{2 \tau} \phi^{S} .
$$

Then Eq. (17) can be rewritten as

$$
\tilde{\phi}_{j}^{n+1}=\tilde{\phi}_{j}^{+, n}-\frac{\Delta t}{\left|V_{j}\right|} \boldsymbol{F}^{n+1 / 2}
$$

where

$$
\tilde{\phi}^{+}=\phi+\frac{\Delta t}{2} \Omega=\frac{2 \tau-\Delta t}{2 \tau+\Delta t} \tilde{\phi}+\frac{2 \Delta t}{2 \tau+\Delta t} \phi^{S} .
$$

It is noted that from the conservative properties of the collision operators given by Eq. (15), we can obtain that

$$
\rho=\int \tilde{g} d \boldsymbol{\xi}, \quad \rho \boldsymbol{u}=\int \boldsymbol{\xi} \tilde{g} d \boldsymbol{\xi}, \quad \rho E=\frac{1}{2} \int\left(\xi^{2} \tilde{g}+\tilde{h}\right) d \boldsymbol{\xi} .
$$

Therefore, in practical computations we can track the distribution function $\tilde{g}$ and $\tilde{h}$ instead of the original ones, which can evolve explicitly according to Eq. (20), provided the microflux $\boldsymbol{F}$ at the cell interface at $t_{n+1 / 2}$ is obtained. In addition to the 
conserved variables, the heat flux $\boldsymbol{q}$ and stress tensor $\boldsymbol{\tau}$ can also be obtained from $\tilde{\phi}$. Actually, it can be shown that

$$
\begin{gathered}
\boldsymbol{q}=\frac{2 \tau}{2 \tau+\Delta t \operatorname{Pr}} \tilde{\boldsymbol{q}}, \quad \text { with } \quad \tilde{\boldsymbol{q}}=\frac{1}{2} \int \boldsymbol{c}\left(c^{2} \tilde{g}+\tilde{h}\right) d \boldsymbol{\xi} . \\
\boldsymbol{\tau}=\frac{2 \tau}{2 \tau+\Delta t} \tilde{\boldsymbol{\tau}}, \quad \text { with } \quad \tilde{\boldsymbol{\tau}}=\int \boldsymbol{c} \boldsymbol{c}\left(\tilde{g}-g^{e q}\right) d \boldsymbol{\xi} .
\end{gathered}
$$

\section{B. Flux evaluation}

The key in evaluating $\boldsymbol{F}^{n+1 / 2}$ is to reconstruct the distribution function $f^{n+1 / 2}$ at the cell interface. To do so we integrate Eq. (16) along the characteristic line within a half time step $s=\Delta t / 2$,

$$
\begin{aligned}
\phi & \left(\boldsymbol{x}_{b}, \boldsymbol{\xi}, t_{n}+s\right)-\phi\left(\boldsymbol{x}_{b}-\boldsymbol{\xi} s, \boldsymbol{\xi}, t_{n}\right) \\
& =\frac{s}{2}\left[\Omega\left(\boldsymbol{x}_{b}, \boldsymbol{\xi}, t_{n}+s\right)+\Omega\left(\boldsymbol{x}_{b}-\boldsymbol{\xi} s, \boldsymbol{\xi}, t_{n}\right)\right],
\end{aligned}
$$

where $\boldsymbol{x}_{b} \in \partial V_{j}$ is the interface center of cell $j$, and the trapezoidal rule is again used to evaluate the collision term. It is noted that the formulation (25) is also implicit due to the collision term $\Omega_{j}^{n+1}$. Similar to the treatment for $\tilde{\phi}$, we introduce another distribution function $\bar{\phi}$ to remove the implicity,

$$
\bar{\phi}=\phi-\frac{s}{2} \Omega=\frac{2 \tau+s}{2 \tau} \phi-\frac{s}{2 \tau} \phi^{S} .
$$

Then Eq. (25) can be rewritten as

$$
\bar{\phi}\left(\boldsymbol{x}_{b}, \boldsymbol{\xi}, t_{n+1 / 2}\right)=\bar{\phi}^{+}\left(\boldsymbol{x}_{b}-\boldsymbol{\xi} s, \boldsymbol{\xi}, t_{n}\right),
$$

where

$$
\bar{\phi}^{+}=\phi+\frac{s}{2} \Omega=\frac{2 \tau-s}{2 \tau+s} \bar{\phi}+\frac{2 s}{2 \tau+s} \phi^{S} .
$$

Therefore, once $\bar{\phi}^{+}\left(\boldsymbol{x}_{b}-\boldsymbol{\xi} s, \boldsymbol{\xi}, t_{n}\right)$ is obtained, the distribution function $\bar{\phi}\left(\boldsymbol{x}_{b}, \boldsymbol{\xi}, t_{n+1 / 2}\right)$ can be determined from Eq. (27). It is noted that the conserved variables can also be obtained from $\bar{g}$ and $\bar{h}$ like Eq. (22),

$$
\rho=\int \bar{g} d \boldsymbol{\xi}, \quad \rho \boldsymbol{u}=\int \boldsymbol{\xi} \bar{g} d \boldsymbol{\xi}, \quad \rho E=\frac{1}{2} \int\left(\xi^{2} \bar{g}+\bar{h}\right) d \boldsymbol{\xi},
$$

which means that $\boldsymbol{W}\left(\boldsymbol{x}_{b}, t_{n+1 / 2}\right)$ can be obtained from $\bar{\phi}\left(\boldsymbol{x}_{b}, \boldsymbol{\xi}, t_{n+1 / 2}\right)$ directly. Furthermore, the heat flux $\boldsymbol{q}\left(\boldsymbol{x}_{b}, t_{n+1 / 2}\right)$ can also be determined from $\bar{\phi}\left(\boldsymbol{x}_{b}, \boldsymbol{\xi}, t_{n+1 / 2}\right)$,

$$
\boldsymbol{q}=\frac{2 \tau}{2 \tau+s \operatorname{Pr}} \overline{\boldsymbol{q}}, \quad \text { with } \quad \overline{\boldsymbol{q}}=\frac{1}{2} \int \boldsymbol{c}\left(c^{2} \bar{g}+\bar{h}\right) d \boldsymbol{\xi}
$$

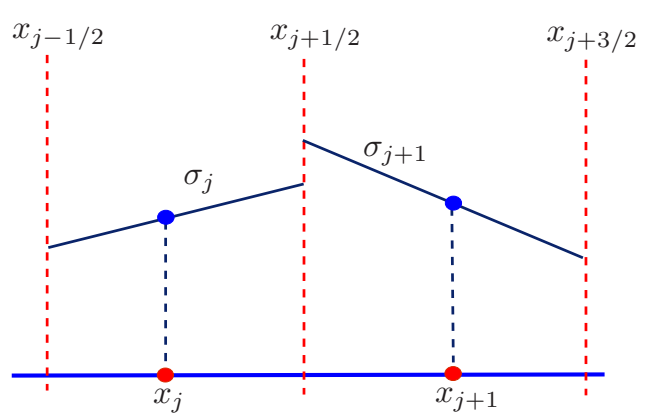

FIG. 1. (Color online) Schematic of 1D cell geometry.

Then the Shakhov distribution $\phi^{S}$ at interface center $\boldsymbol{x}_{b}$ and time $t_{n+1 / 2}$ can be evaluated, and subsequently the original distribution function can be calculated from Eq. (26) as

$$
\begin{aligned}
\phi\left(\boldsymbol{x}_{b}, \boldsymbol{\xi}, t_{n+1 / 2}\right)= & \frac{2 \tau}{2 \tau+s} \bar{\phi}\left(\boldsymbol{x}_{b}, \boldsymbol{\xi}, t_{n}+s\right) \\
& +\frac{s}{2 \tau+s} \phi^{S}\left(\boldsymbol{x}_{b}, \boldsymbol{\xi}, t_{n}+s\right) .
\end{aligned}
$$

Now the task is to determine the $\overline{\boldsymbol{\phi}}^{+}\left(\boldsymbol{x}_{b}-\boldsymbol{\xi} s, \boldsymbol{\xi}, t_{n}\right)$. This is achieved through a reconstruction of the profile of $\bar{\phi}^{+}\left(t_{n}\right)$ in each cell. First, we determine the cell-averaged distribution function $\bar{\phi}^{+}\left(t_{n}\right)$ at the cell center $\boldsymbol{x}_{j}$ from the tracked distribution function $\tilde{\phi}\left(\boldsymbol{x}_{j}, t_{n}\right)$. From Eqs. (19), (26), and (28), we can obtain that

$$
\bar{\phi}^{+}=\frac{2 \tau-s}{2 \tau+\Delta t} \tilde{\phi}+\frac{3 s}{2 \tau+\Delta t} \phi^{S} .
$$

It should be noted that $\tilde{\phi}^{+}$and $\bar{\phi}^{+}$are related. Actually, from Eqs. (21) and (32) we can obtain that

$$
\tilde{\phi}^{+}=\frac{4}{3} \bar{\phi}^{+}-\frac{1}{3} \tilde{\phi}
$$

With this relation the computation can be simplified as noted in the following section.

Assuming that in each cell $\bar{\phi}^{+}$is linear, then we have

$\bar{\phi}^{+}\left(\boldsymbol{x}_{b}-\boldsymbol{\xi} s, \boldsymbol{\xi}, t_{n}\right)=\bar{\phi}^{+}\left(\boldsymbol{x}_{j}, \boldsymbol{\xi}, t_{n}\right)+\left(\boldsymbol{x}_{b}-\boldsymbol{x}_{j}-\boldsymbol{\xi} s\right) \cdot \boldsymbol{\sigma}_{j}$,

$$
\left(\boldsymbol{x}_{b}-\boldsymbol{\xi} s\right) \in V_{j}
$$

where $\sigma_{j}$ is the slope of $\bar{\phi}^{+}$in cell $j$. As an example, in Fig. 1 a one-dimensional (1D) case is shown. In this case, in order to reconstruct the distribution function $\phi$ at the cell interface $x_{b}=x_{j+1 / 2}$, the distribution function $\bar{\phi}^{+}$is approximated as

$$
\bar{\phi}^{+}\left(x_{b}-\xi s, \xi, t_{n}\right)= \begin{cases}\bar{\phi}^{+}\left(x_{j}, \xi, t_{n}\right)+\left(x_{b}-\xi s-x_{j}\right) \sigma_{j}, & \xi>0, \\ \bar{\phi}^{+}\left(x_{j+1}, \xi, t_{n}\right)+\left(x_{b}-\xi s-x_{j+1}\right) \sigma_{j+1}, & \xi<0 .\end{cases}
$$

The slope $\sigma_{j}$ in each cell can be reconstructed from the cell-averaged values using some numerical limiters. For example, in the $1 \mathrm{D}$ case shown in Fig. 1, we can use the van Leer limiter [20], i.e.,

$$
\sigma_{j}=\left[\operatorname{sgn}\left(s_{1}\right)+\operatorname{sgn}\left(s_{2}\right)\right] \frac{\left|s_{1}\right|\left|s_{2}\right|}{\left|s_{1}\right|+\left|s_{2}\right|},
$$

where

$$
s_{1}=\frac{\bar{\phi}_{j}^{+}-\bar{\phi}_{j-1}^{+}}{x_{j}-x_{j-1}}, \quad s_{2}=\frac{\bar{\phi}_{j+1}^{+}-\bar{\phi}_{j}^{+}}{x_{j+1}-x_{j}} .
$$




\section{Evolution procedure}

In summary, in the procedure of the DUGKS the flow evolution from time step $t_{n}$ to $t_{n+1}$ can be summarized in the following (assuming $\boldsymbol{x}_{b}$ is the interface center of cell $j$ centered at $\boldsymbol{x}_{j}$ ):

(1) Calculate the microflux $\boldsymbol{F}$ at cell interface $\boldsymbol{x}_{b}$ and at time $t_{n+1 / 2}$.

(a) Calculate $\bar{\phi}^{+}$from $\tilde{\phi}$ at each cell center with velocity $\boldsymbol{\xi}$ according to Eq. (32);

(b) Reconstruct the gradient of $\bar{\phi}^{+}$(i.e., $\sigma$ ) in each cell using certain numerical limiters, e.g., Eq. (36) in 1D case;

(c) Reconstruct the distribution function $\bar{\phi}^{+}$at $\boldsymbol{x}_{b}-\boldsymbol{\xi} s$ according to Eq. (34);

(d) Determine the distribution function $\bar{\phi}$ at cell interface at time $t_{n+1 / 2}$ according to Eq. (27);

(e) Calculate the conservative flow variables $\boldsymbol{W}\left(\boldsymbol{x}_{b}, t_{n+1 / 2}\right)$ and heat flux $\boldsymbol{q}\left(\boldsymbol{x}_{b}, t_{n+1 / 2}\right)$ from $\bar{\phi}\left(\boldsymbol{x}_{b}, \boldsymbol{\xi}, t_{n+1 / 2}\right)$ [see Eqs. (29) and (30)];

(f) Calculate the original distribution function $\phi$ at cell interface and $t_{n+1 / 2}$ from $\bar{\phi}\left(\boldsymbol{x}_{b}, \boldsymbol{\xi}, t_{n+1 / 2}\right)$ and $\phi^{S}\left(\boldsymbol{x}_{b}, \boldsymbol{\xi}, t_{n+1 / 2}\right)$ according to Eq. (31);

(g) Calculate the microflux $\boldsymbol{F}^{n+1 / 2}$ through each cell interface from $\phi^{n+1 / 2}$ according to Eq. (18).

(2) Calculate $\tilde{\phi}^{+}$at cell center and time $t_{n}$ according to Eq. (33).

(3) Update the cell-averaged $\tilde{\phi}$ in each cell from $t_{n}$ to $t_{n+1}$ according to Eq. (17).

The particle velocity $\boldsymbol{\xi}$ is continuous in the above procedure. In practical computations, the velocity space will be discretized into a set of discrete velocities $\boldsymbol{\xi}_{i}(i=1,2, \ldots, b)$. Usually the discrete velocity set is chosen as the abscissas of certain quadrature rules such as the Gaussian-Hermite or NewtonCotes formula, and the integrals in the above procedure will be replaced by the quadrature. For example, the conservative variables can be computed as

$$
\begin{aligned}
\rho & =\sum_{i=1}^{b} w_{i} \tilde{g}\left(\boldsymbol{\xi}_{i}\right), \quad \rho \boldsymbol{u}=\sum_{i=1}^{b} w_{i} \boldsymbol{\xi}_{i} \tilde{g}\left(\boldsymbol{\xi}_{i}\right), \\
\rho E & =\frac{1}{2} \sum_{i=1}^{b} w_{i}\left[\xi^{2} \tilde{g}\left(\boldsymbol{\xi}_{i}\right)+\tilde{h}(\boldsymbol{\xi})\right],
\end{aligned}
$$

where $w_{i}$ is the associate quadrature weights.

\section{ANALYSIS OF DUGKS}

We now discuss some important properties of the DUGKS. First, we will show the DUGKS has the asymptotic preserving (AP) property [14,21], namely, (i) the time step $\Delta t$ is independent of the particle collision time for all Knudsen numbers, and (ii) the scheme is consistent with the NavierStokes equations in the continuum limit. Regarding the time step, it is noted that the particle transport and collisions are coupled in the reconstruction of the interface distribution function, which is necessary for an AP scheme [14]. This coupling also releases the constraint on the collision time and the time step as in the operator-splitting schemes, and the time step can be determined by the Courant-Friedrichs-Lewy (CFL) condition $[14,17]$,

$$
\Delta t=\alpha \frac{\Delta x}{U_{m}+\xi_{m}},
$$

where $\alpha$ is the CFL number, $\Delta x$ is the minimal grid spacing, $\xi_{m}$ is the maximum discrete velocity, and $U_{m}$ is the maximum flow velocity. $\Delta t$ determined in this way does not depend on the relaxation time $\tau$, and the DUGKS is uniformly stable with respect to the Knudsen number.

Regarding point (ii), it is noted that in the continuum limit as $\tau \ll \Delta t$, the distribution function in a cell given by Eq. (34) can be approximated as

$\bar{\phi}^{+}\left(\boldsymbol{x}_{b}-\boldsymbol{\xi} s, \boldsymbol{\xi}, t_{n}\right)=\bar{\phi}^{+}\left(\boldsymbol{x}_{b}, \boldsymbol{\xi}, t_{n}\right)-s \boldsymbol{\xi} \cdot \boldsymbol{\sigma}_{b}+O\left(\Delta x^{2}\right)$,

where $\boldsymbol{\sigma}_{b}$ is the slope of $\bar{\phi}^{+}\left(\boldsymbol{\xi}, t_{n}\right)$ at the cell interface $\boldsymbol{x}_{b}$. Furthermore, following the procedure given in Appendix B of Ref. [17], we can show that

$$
\phi\left(\boldsymbol{x}_{b}, \boldsymbol{\xi}, t\right)=\phi^{S}\left(\boldsymbol{x}_{b}, \boldsymbol{\xi}, t\right)-\tau D_{t} \phi^{S}\left(\boldsymbol{x}_{b}, \boldsymbol{\xi}, t\right)+O\left(\partial^{2}\right),
$$

$$
\phi^{S}\left(\boldsymbol{x}_{b}, \boldsymbol{\xi}, t_{n}+s\right)=\phi^{S}\left(\boldsymbol{x}_{b}, \boldsymbol{\xi}, t_{n}\right)+s \partial_{t} \phi^{S}\left(\boldsymbol{x}_{b}, \boldsymbol{\xi}, t_{n}\right)+O\left(\partial^{2}\right) \text {. }
$$

Then, with the aids of these results, we can obtain from Eqs. (27), (28), and (31) that (refer to Appendix B of Ref. [17])

$$
\begin{aligned}
\phi\left(\boldsymbol{x}_{b}, \boldsymbol{\xi}, t_{n}+s\right) \approx & \phi^{S}\left(\boldsymbol{x}_{b}, \boldsymbol{\xi}, t_{n}\right)-\tau\left(\partial_{t}+\boldsymbol{\xi} \cdot \nabla\right) \phi^{S}\left(\boldsymbol{x}_{b}, \boldsymbol{\xi}, t_{n}\right) \\
& +s \partial_{t} \phi^{S}\left(\boldsymbol{x}_{b}, \boldsymbol{\xi}, t_{n}\right)
\end{aligned}
$$

which recovers the Chapman-Enskog approximation for the Navier-Stokes solution [14,22]. This fact suggests that the DUGKS can be viewed as a Navier-Stokes solver in the continuum limit. It is also noted that the use of the midpoint and trapezoidal rules in Eqs. (17) and (25), as well as the linear reconstruction of the distribution function at the cell interface, ensures a second-order accuracy in both space and time in the continuum limit.

On the other hand, in the free-molecule limit where $\tau \gg$ $\Delta t=2 s$, we can find from Eq. (28) that $\bar{\phi}^{+}\left(\boldsymbol{x}_{b}-\boldsymbol{\xi} s, \boldsymbol{\xi}, t_{n}\right) \approx$ $\bar{\phi}\left(\boldsymbol{x}_{b}-\boldsymbol{\xi} s, \boldsymbol{\xi}, t_{n}\right)$, and then from Eq. (27) that $\bar{\phi}\left(\boldsymbol{x}_{b}, \boldsymbol{\xi}, t_{n}+\right.$ $s)=\overline{\boldsymbol{\phi}}^{+}\left(\boldsymbol{x}_{b}-\boldsymbol{\xi} s, \boldsymbol{\xi}, t_{n}\right) \approx \bar{\phi}\left(\boldsymbol{x}_{b}-\boldsymbol{\xi} s, \boldsymbol{\xi}, t_{n}\right)$. Furthermore, the relationship between $\bar{\phi}$ and $\phi$ as shown in Eq. (26) gives that $\phi\left(\boldsymbol{x}_{b}, \boldsymbol{\xi}, t_{n}+s\right) \approx \bar{\phi}\left(\boldsymbol{x}_{b}, \boldsymbol{\xi}, t_{n}+s\right) \approx \phi\left(x_{b}-\boldsymbol{\xi} s, t_{n}\right)$, which is just the collisionless limit.

We now point out some key differences between the present DUGKS and the UGKS [14,15], which is also designed for all Knudsen number flows, although both share many common features such as the multidimensional nature, AP property, and the coupling of particle transport and collision. The first key difference is that the cell-averaged conservative variables $\boldsymbol{W}$ and heat flux $\boldsymbol{q}$ in each cell are required to evolve along with the cell-averaged distribution functions in the UGKS, because the collision term is discretized with the trapezoidal rule and the evaluation of the implicit part needs these quantities. However, with the newly introduced distribution function $\tilde{\phi}$, the implicity in the collision term is removed in the DUGKS, and $\boldsymbol{W}$ and $\boldsymbol{q}$ are not required to evolve. The second key difference between DUGKS and UGKS lies in the reconstruction of the distribution function at cell interfaces. In the UGKS $[14,15]$, the interface distribution function $\phi\left(\boldsymbol{x}_{b}, t\right)$ 
is constructed based on the integral solution of the kinetic equation with certain approximations, while in the present DUGKS it is constructed based on the characteristic solution, which is much simpler. The third difference is that the DUGKS is solely based on the single relaxation kinetic model due to its combination of the distribution function and the collision term, but the UGKS can be extended to the full Boltzmann collision term as well [23]. Despite these differences, we will show in the next section that the present DUGKS can yield numerical predictions nearly the same as the UGKS.

Finally, we make some discussions about the computational cost of the DUGKS in comparison with several other wellaccepted kinetic methods, i.e., the UGKS, DSMC, and lattice Boltzmann equation (LBE) methods. (i) As compared with the UGKS, the DUGKS is expected to be faster because of the differences in the evolution of the distribution functions at cell interfaces as discussed above. Both need nearly identical computational memory, since they use the same number of grid points in particle velocity space. The numerical tests given below support this point. (ii) Regarding the DSMC method, which is a stochastic particle method that decouples the particle movement and collision process, the time step and cell size are required to be smaller than the collision time and mean free path, respectively, and its computational cost is linearly proportional to the number of simulated particles [24]. Therefore, for highly rarefied high-speed flows the computational efficiency of DSMC can be higher than the DUGKS with far smaller memory requirement; but for near continuum low-speed flows the computational efficiency of DSMC can be much lower than DUGKS. The statistical noise in DSMC makes it impracticable for low-speed flows where the flow velocity is far smaller than the sound speed. On the other hand, the DUGKS has no such limitation for low-speed and near continuum flows. It is noted that a variety of improved DSMC methods for reducing the statistical noise have been developed from different viewpoints [e.g., 25,26], but the fundamental requirements for the kinetic scale time step and cell size still limit their applications for the near continuum flows. (iii) For the LBE method, a regular lattice (or mesh) associated with the underlying discrete velocity set is usually adopted [27], which severely limits its applications for flows with large gradient. A recent numerical comparative study [28] between the isothermal DUGKS and LBE indicates that with the same uniform mesh, the LBE can be about 4 times faster than DUGKS for low-Reynolds-flow simulations. But, the numerical stability of DUGKS is much better than LBE with the same uniform mesh. For example, for the two-dimensional (2D) lid-driven cavity flow the maximum Reynolds number at which the LBE is stable is about 1900 on a $128 \times 128$ mesh, while the computation of the DUGKS is still stable even at $\operatorname{Re}=10^{5}$ [28]. Furthermore, the DUGKS can be much more efficient than LBE for high-Reynoldsnumber flows with an adaptation of nonuniform mesh. The performance differences between the present DUGKS and thermal LBE are expected to be similar, since their structures in both schemes are the same as the isothermal counterparts.

\section{NUMERICAL TESTS}

The present DUGKS will be validated by a number of test problems in different flow regimes in this section. The problems include 1D and 2D subsonic or supersonic flows. In the simulations the van Leer limiter [20] is used in the reconstruction of the interface distribution function.

\section{A. 1D shock structure}

The first test case is the argon shock structure from low to high Mach numbers. The results of the present DUGKS simulations will be compared with the Boltzmann solution, DSMC result, and UGKS prediction. The densities, velocities, and temperatures at upstream $\left(\rho_{1}, u_{1}, T_{1}\right)$ and downstream $\left(\rho_{2}, u_{2}, T_{2}\right)$ satisfy the Rankine-Hugoniot conditions [29]. The Prandtl number and specific heat ratio for argon are $\operatorname{Pr}=2 / 3$ and $\gamma=5 / 3$, respectively, and the viscosity depends on the temperature, $\mu \propto T^{w}$, where $w$ relates to the intermolecular interactions [29]. The mean free path $\lambda$ is related to the viscosity as [24]

$$
\lambda=\frac{2 \mu(7-2 w)(5-2 w)}{15 \rho(2 \pi R T)^{1 / 2}} .
$$

In the simulations the flow variables are normalized by the corresponding upstream quantities, and the characteristic density, length, velocity, and time are chosen to be $\rho_{1}, \lambda_{1}$, $\sqrt{2 R T_{1}}$, and $\lambda_{1} / \sqrt{2 R T_{1}}$, respectively. Specifically, in our simulations the upstream quantities are set to be $\rho_{1}=1.0$, $\lambda_{1}=1.0$, and $T_{1}=1.0$, and the velocity $u_{1}$ is determined from the upstream Mach number Ma $=u_{1} / \sqrt{\gamma R T_{1}}$ specified case by case. The reference viscosity is set to be $\mu_{0}=0.5539$, and the downstream quantities are then determined from the Rankine-Hugoniot conditions:

$$
\begin{aligned}
\mathrm{Ma}^{\prime} & =\sqrt{\frac{\mathrm{Ma}^{2}(\gamma-1)+2}{2 \gamma \mathrm{Ma}^{2}-(\gamma-1)}}, \\
\frac{\rho_{2}}{\rho_{1}} & =\frac{(\gamma+1) \mathrm{Ma}^{2}}{(\gamma-1) \mathrm{Ma}^{2}+2}, \\
\frac{T_{2}}{T_{1}} & =\frac{\left(1+\frac{\gamma-1}{2} \mathrm{Ma}^{2}\right)\left(\frac{2 \gamma}{\gamma-1} \mathrm{Ma}^{2}-1\right)}{\mathrm{Ma}^{2}\left(\frac{2 \gamma}{\gamma-1}+\frac{\gamma-1}{2}\right)},
\end{aligned}
$$

where $\mathrm{Ma}^{\prime}$ is the downstream Mach number, from which the downstream velocity $u_{2}$ can be determined.

The computational domain is chosen to be $-25 \lambda_{1} \leqslant x \leqslant$ $25 \lambda_{1}$. A uniform mesh with 100 cells is used so that the mesh space is $\Delta x=0.5 \lambda_{1}$. With this mesh, the shock structure can be well resolved and the numerical results are found to be grid independent. The discrete velocity set is determined by the Newton-Cotes quadrature with 101 points distributed uniformly in $[-15,15]$. Initially, the distribution functions at $x \leqslant 0$ are set to be the Maxwellian distribution with the upstream state, and those at $x>0$ are set to be the Maxwellian distribution with the downstream state. The CFL number used in all simulations is set to be 0.95 .

First we consider the hard-sphere model (i.e., $w=0.5$ ), which was also studied by Ohwada by solving the full Boltzmann equation numerically [30], and by $\mathrm{Xu}$ and Huang using the UGKS method with the Shakhov model [31]. In Fig. 2 the profiles of the normalized density $\rho^{*}=\rho / \rho_{1}$, temperature $T^{*}=T / T_{1}$, heat flux $q_{x}^{*}=q_{x} / p_{1}$, and shear stress $\tau_{x x}^{*}=\tau_{x x} / p_{1}\left(2 R T_{1}\right)^{3 / 2}$ with $p_{1}=\rho_{1} R T_{1}$ are shown at 

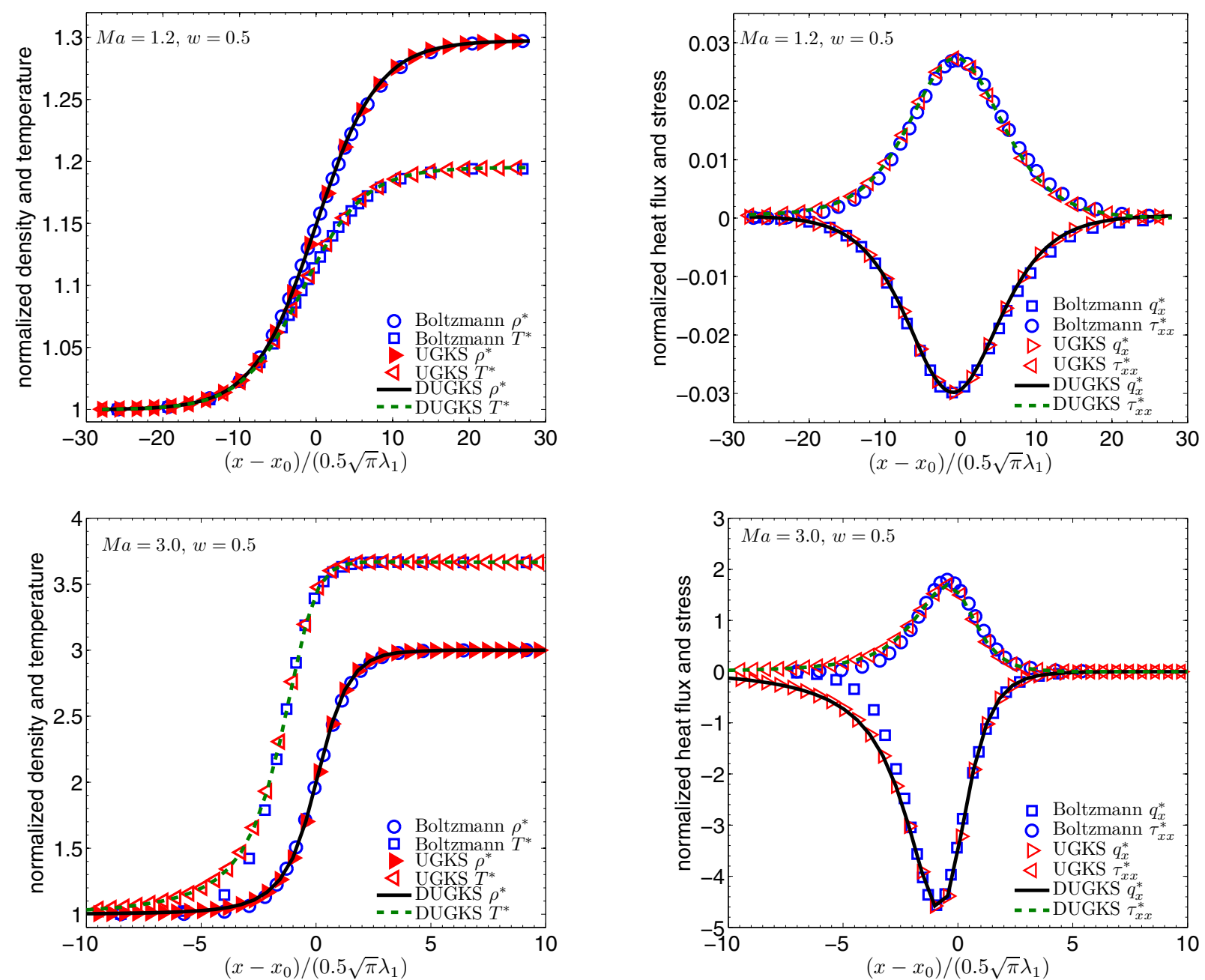

FIG. 2. (Color online) Shock structure of hard-sphere gas at different Mach numbers $\left(\mu_{0}=0.5539\right)$ : (left) density $\left(\rho^{*}\right)$ and temperature $\left(T^{*}\right)$; (right) stress $\left(\tau_{x x}^{*}\right)$ and heat flux $\left(q_{x}^{*}\right)$.

$\mathrm{Ma}=1.2$ and 3.0. Here the location of the shock is chosen to be $x_{0}$ such that $\rho\left(x_{0}\right)=\left(\rho_{1}+\rho_{2}\right) / 2$; the heat flux and stress are computed according to Eqs. (23) and (24). The results are compared with those of the Boltzmann and UGKS solutions. It can be seen that the density, temperature, shear stress, and heat flux predicted by the present DUGKS all agree well with the UGKS and Boltzmann solutions at $\mathrm{Ma}=1.2$. In the case of $\mathrm{Ma}=3.0$, the results of DUGKS agree well with those of the UGKS, and the density and stress of both methods agree well with the Boltzmann data, but clear deviations are observed in the temperature and heat flux profiles in the upstream region. Specifically, the temperature profiles predicted by the DUGKS and the UGKS methods both rise early in the upstream region in comparison with the Boltzmann solution, and the predicted heat flux profiles decrease early accordingly. These deviations are due to the use of a single relaxation time in the Shakhov collision model employed in the DUGKS and UGKS methods, which may be reduced by using more complicated collision models, such as the velocity-dependent relaxation model or the full Boltzmann model [23,32]. However, the use of other collision operators will lead to additional computational cost. It is emphasized here that the difference between the present DUGKS and the UGKS [31], which is accepted as a well-established efficient method for flows ranging from free-molecule to continuum regimes, are rather small. For instance, at $\mathrm{Ma}=3.0$ the maximum relative differences in density and temperature between the two solutions are about $8.09 \times 10^{-5}$ and $2.24 \times 10^{-4}$, respectively.

We next test the shock structure of Ma $=8$ with $w=0.68$ as studied by the DSMC [33] and UGKS [31] methods. In Fig. 3 the normalized density $\rho^{\prime}=\left(\rho-\rho_{1}\right) /\left(\rho_{2}-\rho_{1}\right)$, temperature $T^{\prime}=\left(T-T_{1}\right) /\left(T_{2}-T_{1}\right)$, heat flux $q_{x}^{*}$, and stress $\tau_{x x}^{*}$ are shown and compared with the DSMC and UGKS data [31,33]. Again the results predicted by the present DUGKS are in close agreement with those of the UGKS method, and both compare well with the DSMC results in the downstream region. However, at this high Mach number the temperature and the heat flux profiles predicted by the DUGKS and UGKS methods show even larger deviations in the upstream region in comparison with those of the DSMC. The Mach-number-dependent discrepancies in temperature and heat flux can again be attributed to the relaxation approximation of the collision operator [24]. On the other hand, it is noted that the shear stress $\tau_{x x}$ predicted by the 

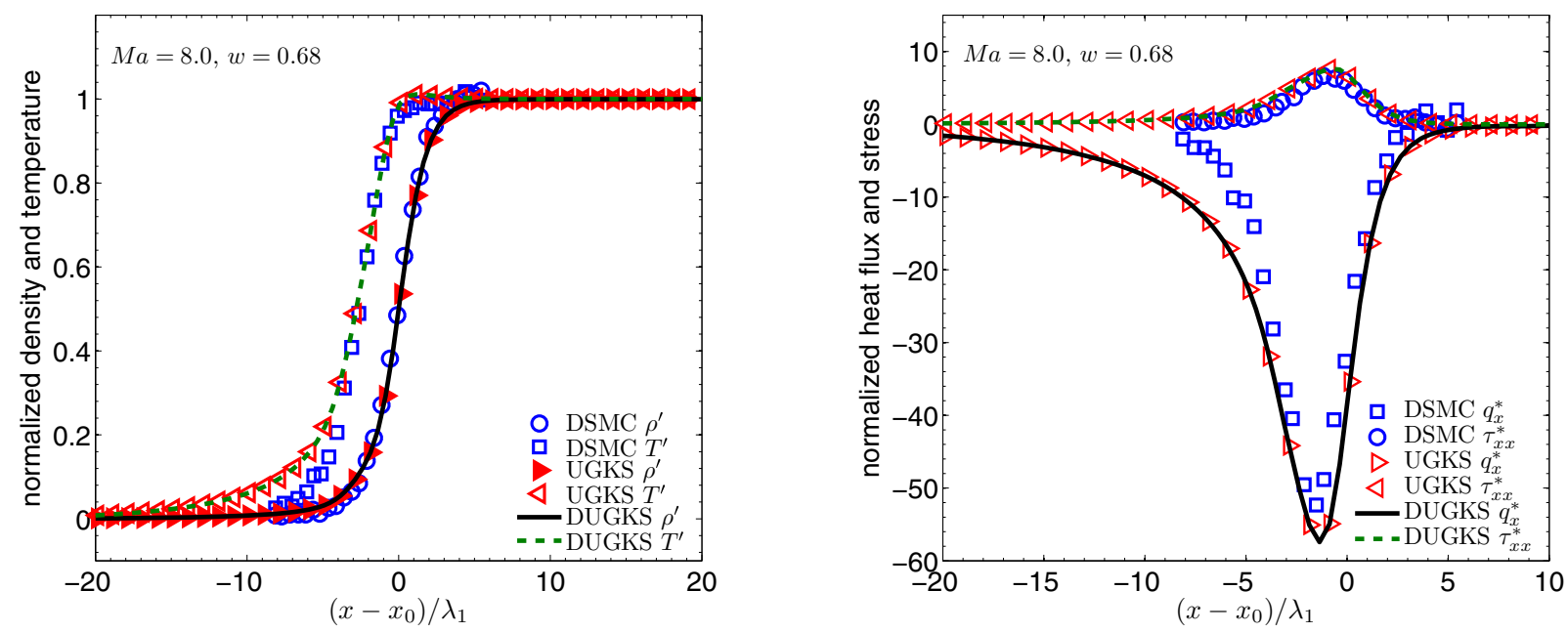

FIG. 3. (Color online) Shock structure with Ma $=8$ and $w=0.68\left(\mu_{0}=0.5539\right)$ : (left) density $\left(\rho^{\prime}\right)$ and temperature $\left(T^{\prime}\right)$; (right) stress $\left(\tau_{x x}^{*}\right)$ and heat flux $\left(q_{x}^{*}\right)$.

present DUGKS agrees well with the DSMC result, and the peak values of the heat flux predicted by the three methods are quite close.

The present DUGKS is also tested as a shock-capturing scheme. This is achieved by varying the cell size $\Delta x$ with a fixed CFL number, as suggested in [14]. As an example, the $\mathrm{Ma}=1.2$ shock structure of a hard-sphere gas with a fixed upstream mean free path $\left(\lambda_{1}=1.0\right)$ is simulated by the present DUGKS. In the calculations, the cell size $\Delta x$ changes from $0.5 \lambda_{1}$ to $100 \lambda_{1}$, and the CFL number is fixed at 0.95 so that the time step changes with the cell size accordingly. In Fig. 4 the density and temperature profiles are shown with different cell sizes. As observed, the solution goes from a well resolved to highly under-resolved solution with increasing of cell sizes. Particularly, as $\Delta x=100 \lambda_{1}$ the solution agrees very well with the exact solution of the Euler equations, suggesting that the DUGKS becomes an effective shock-capturing scheme in this case. The low dissipative nature of the DUGKS is due to the coupling of collision and transport in the reconstruction of the

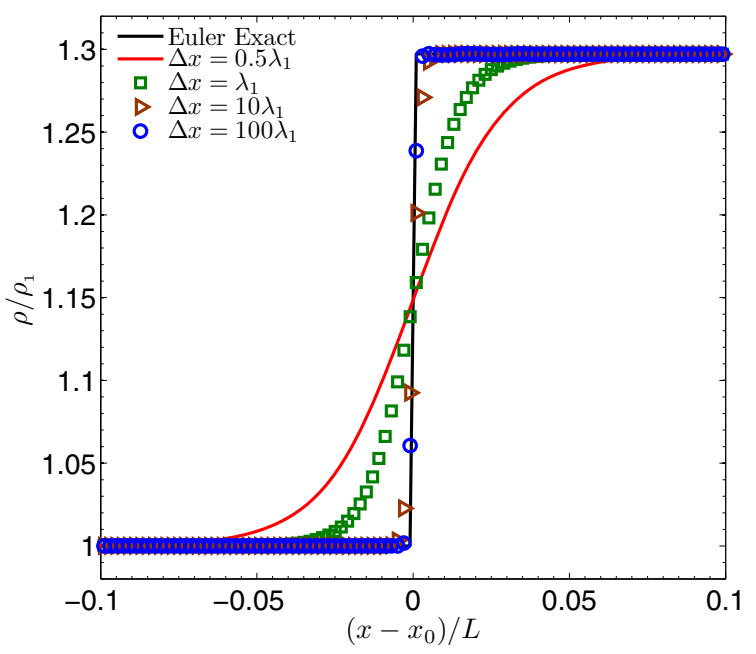

cell interface flux. It is noted that the discrete ordinate method (DOM) may encounter numerical instability with $\Delta x=100 \lambda_{1}$ and a CFL number 0.95 [14], and a much smaller CFL number should be used to obtain a stable but more dissipative solution.

\section{B. Shock tube}

The second test case is the standard Sod's shock tube problem [34]. The computational domain is $-0.5 \leqslant x \leqslant 0.5$, and initially the density, velocity, and pressure are set to be

$$
(\rho, u, p)= \begin{cases}\left(\rho_{1}, u_{1}, p_{1}\right)=(1.0,0.0,1.0) & x \leqslant 0 \\ \left(\rho_{2}, u_{2}, p_{2}\right)=(0.125,0.0,0.1) & x>0\end{cases}
$$

The gas considered is modeled as hard-sphere molecules such that the viscosity is determined as $\mu=\mu_{0}\left(T / T_{0}\right)^{0.5}$, where $\mu_{0}$ is the reference viscosity at reference temperature $T_{0}$. The reference mean free path $\lambda_{0}$ is then changed by adjusting

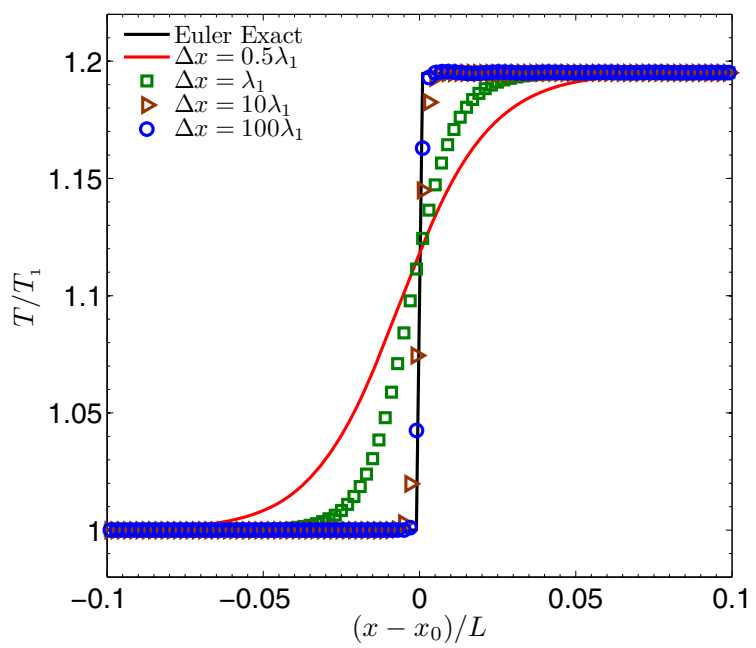

FIG. 4. (Color online) Density and temperature profiles of the shock structure (Ma $\left.=1.2, w=0.5, \mu_{0}=0.5539\right)$ with different cell sizes and CFL number 0.95 . 

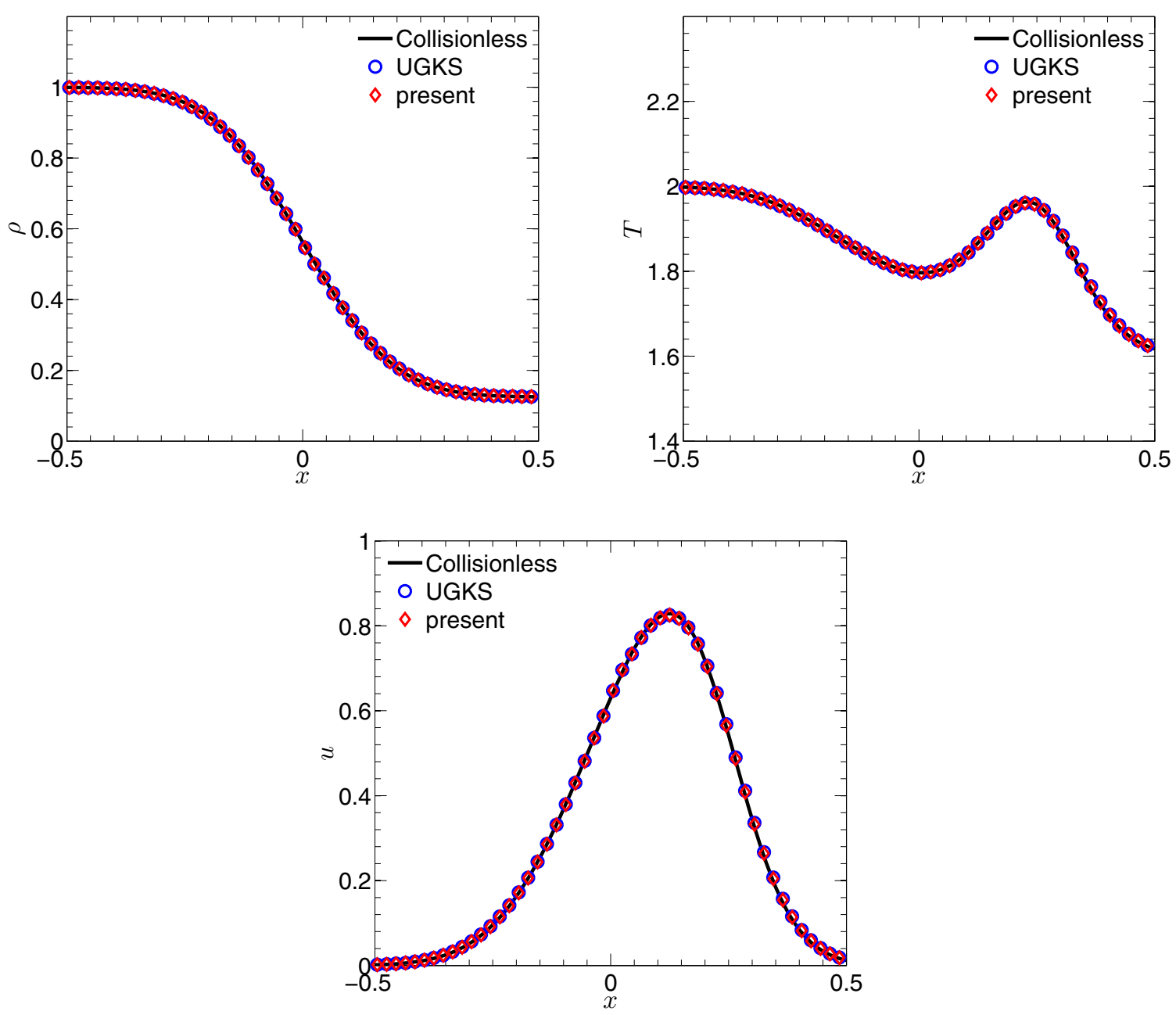

FIG. 5. (Color online) Density, temperature, and velocity profiles of the shock tube test $\left(\mu_{0}=10.0\right)$.

the $\mu_{0}$,

$$
\lambda_{0}=\frac{16}{5} \frac{\mu_{0}}{p_{0}} \sqrt{\frac{R T_{0}}{2 \pi}}
$$

where $p_{0}$ is the reference pressure. Here we take $\rho_{1}, p_{1}$, and $T_{1}$ as the reference density, pressure, and temperature, respectively. With different $\mu_{0}$, the flow will have different degree of rarefaction, which can be used to test the capability of the DUGKS method for simulating flows in different regimes.

In the computation a uniform grid with 100 cells is used to cover the physical domain, and 201 discrete velocities uniformly distributed in $[-10,10]$ are used to discretize the velocity space, and the Newton-Cotes quadrature is used to evaluate the velocity moments. The CFL number is set to be 0.95 in all simulations, and the output time is $t=0.15$. In all cases the internal freedom is set to be $K=2$ so that the ratio of specific heats is $\gamma=1.4$. In order to make a comparison with the UGKS, $\mu_{0}$ changes from 10 to $10^{-5}$ as in Ref. [14], such that the flow ranges from continuum to free-molecular regimes.

Figure 5 shows the density, temperature, and velocity profiles as $\mu_{0}=10.0$, as well as the UGKS results and the solution of the collisionless Boltzmann equation (see Appendix A). In this case the corresponding Knudsen number at the left boundary is about 12.77 and the flow falls in the free-molecular regime. It can be seen that the DUGKS results agree excellent with the collisionless Boltzmann solution and the UGKS data. As $\mu_{0}$ decreases to 0.1 , the flow falls in the slip regime. The result of the DUGKS in this case is shown in Fig. 6 and compared with the solution of the UGKS method. The collisionless Boltzmann solution is also included as a reference to demonstrate the collision effect for slip flows. The results of the DUGKS and UGKS are nearly identical, and there are some clear deviations from the collisionless Boltzmann solutions, which is not surprising since collision effects are significant in such case.

The results for $\mu_{0}=10^{-5}$ are shown in Fig. 7, where the exact solution of the Euler equations, the results of the BGK scheme for the Navier-Stokes equations (BGK-NS) [22], and the results of the UGKS scheme are shown together. In this case the flow is in the continuum regime and the DUGKS becomes a shock-capturing scheme. It can be seen that the DUGKS results agree well with those of the BGK-NS and UGKS methods, but some deviations from the Euler solution are observed. The disparity is due to the fact that the interparticle collision effect is considered in the DUGKS, which means that the DUGKS solves the Navier-Stokes equations rather than the Euler ones in this case. Actually, the DUGKS acts like a shock-capturing method, and the numerical dissipation in 

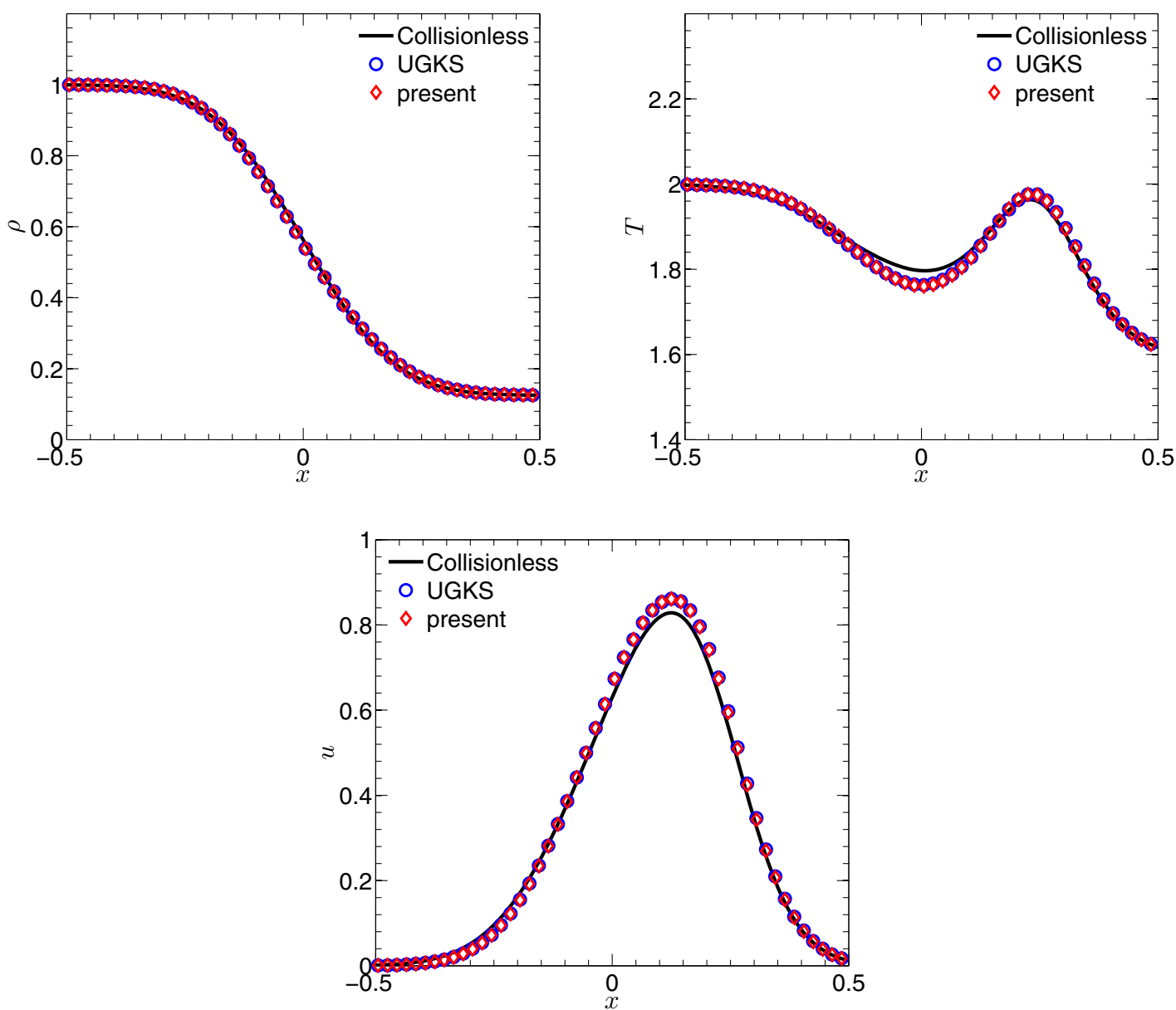

FIG. 6. (Color online) Density, temperature, and velocity profiles of the shock tube test $\left(\mu_{0}=0.1\right)$.

the discontinuous region plays an important role. The detailed results are sensitive to the limiters. We note that in many other shock-capturing methods based on the Euler equations, the reconstruction is based on the characteristic variables instead of the conservative ones in order to remove this kind of overor undershoot. The agreement between the DUGKS results and those of the BGK-NS method, which is a Navier-Stokes solver [22], also confirms this point. The collision effect is also considered in the UGKS method [14], and so it is not surprising that similar features are observed in the numerical results. We also note that numerical oscillation appears at the contact wave, which may come from the numerical limiter in the reconstruction of flow variables at cell interfaces [14].

The above results suggest that the DUGKS and UGKS give almost identical predications from continuum to freemolecular flows. It is interesting to make a comparison between their computational costs. A number of tests with different meshes and discrete velocity sets are carried out, and it is found that generally the CPU time of the DUGKS is about $70 \%$ of that of the UGKS. For instance, with a mesh of 100 cells and 101 discrete velocities, the CPU times for 10000 iterations of the DUGKS and the UGKS are 9.940 and 14.248 $\mathrm{s}$, respectively.

\section{Two-dimensional Riemann problem}

We now test the unified property of the DUGKS with the 2D Riemann problem with constant initial data in each quadrant. The solution of the Euler equations for this problem can have a number of different configurations with different initial setups, and a variety of numerical studies have been reported in the past two decades [35-40]. Here we choose one of the typical configurations as listed in Ref. [39], where the initial condition is given by

$$
(\rho, u, v, p)=\left\{\begin{array}{lll}
\left(\rho_{1}, u_{1}, v_{1}, p_{1}\right)=(0.5313,0,0,0.4), & x>0, & y>0, \\
\left(\rho_{2}, u_{2}, v_{2}, p_{2}\right)=(1,0.7276,0,1), & x \leqslant 0, & y>0, \\
\left(\rho_{3}, u_{3}, v_{3}, p_{3}\right)=(0.8,0,0,1), & x \leqslant 0, & y \leqslant 0, \\
\left(\rho_{4}, u_{4}, v_{4}, p_{4}\right)=(1,0,0.7276,1), & x>0, & y \leqslant 0 .
\end{array}\right.
$$



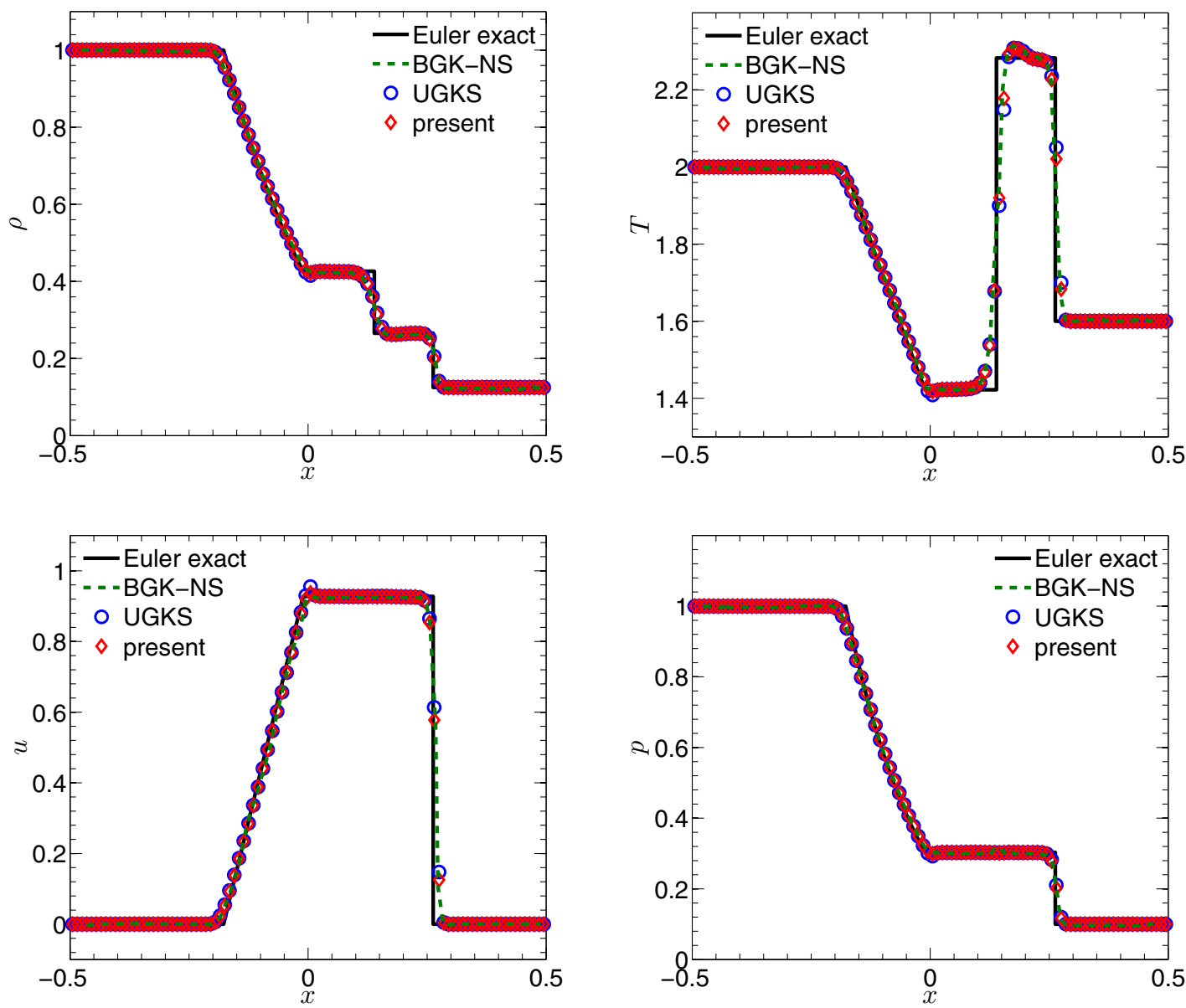

FIG. 7. (Color online) Density, temperature, velocity, and pressure profiles of the shock tube test $\left(\mu_{0}=10^{-5}\right)$.

In our simulations, we set $\operatorname{Pr}=2 / 3$ and $\gamma=1.4$. A $400 \times 400$ uniform mesh is employed to discretize the physical domain $0 \leqslant x, y \leqslant 1$, and the CFL number is set to be 0.5 in all simulations. As in the 1D shock tube test, a reference viscosity $\mu_{0}$ at reference temperature $T_{0}$ is employed to characterize the rarefaction of the gas, and the local viscosity is determined by $\mu=\mu_{0}\left(T / T_{0}\right)^{w}$ with $w=0.5$. At the four boundaries the boundary conditions are set to be $\partial_{\boldsymbol{n}} f=0$, where $\boldsymbol{n}$ is the outward unit normal vector.

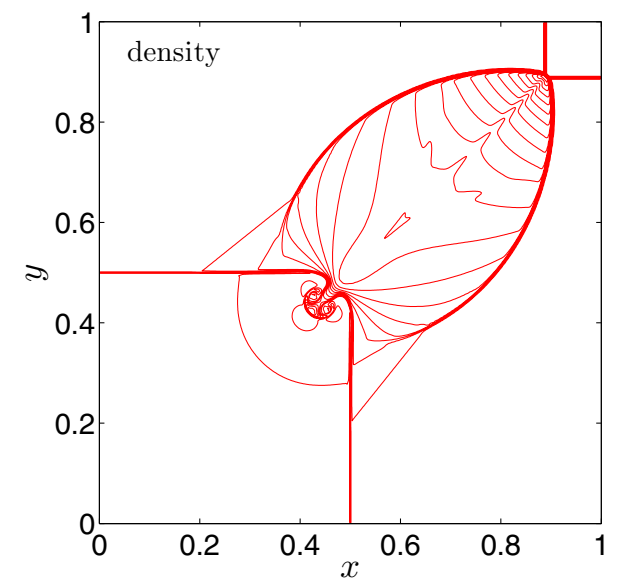

We first present the results as $\mu_{0}=10^{-7}$. The reference mean free path $\lambda_{0}=\left(\mu_{0} / p_{0}\right) \sqrt{\pi R T_{0} / 2}$ and the collision time $\tau=\mu_{0} / p_{0}$ are both in the order of $10^{-7}$, and the flow is in the continuum regime. In the simulation a $8 \times 8$ discrete velocity set based on the half-range Gauss-Hermite quadrature [41] is employed. The density and temperature contours at $t=0.25$ are shown in Fig. 8. It is clear that in this case the DUGKS becomes a shock capture scheme, since now $\Delta x=2.5 \times 10^{-3} \gg \lambda_{0}$ and $\Delta t \sim 10^{-4} \gg \tau$. The density

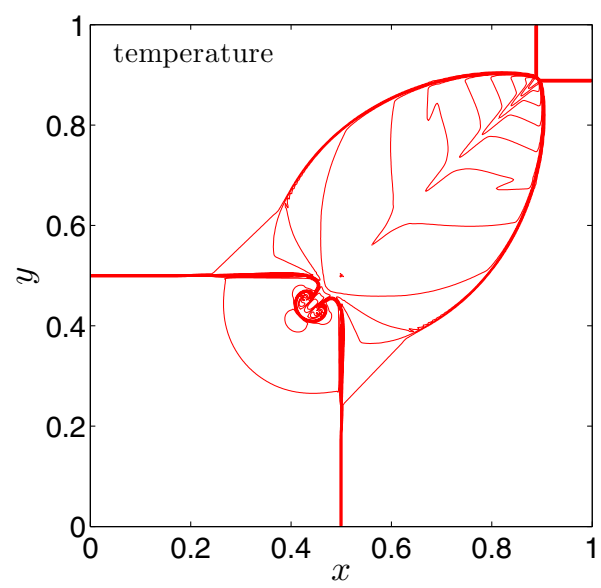

FIG. 8. (Color online) Density and temperature distributions of the 2D Riemann problem $\left(\mu_{0}=10^{-7}\right)$. 
(a)

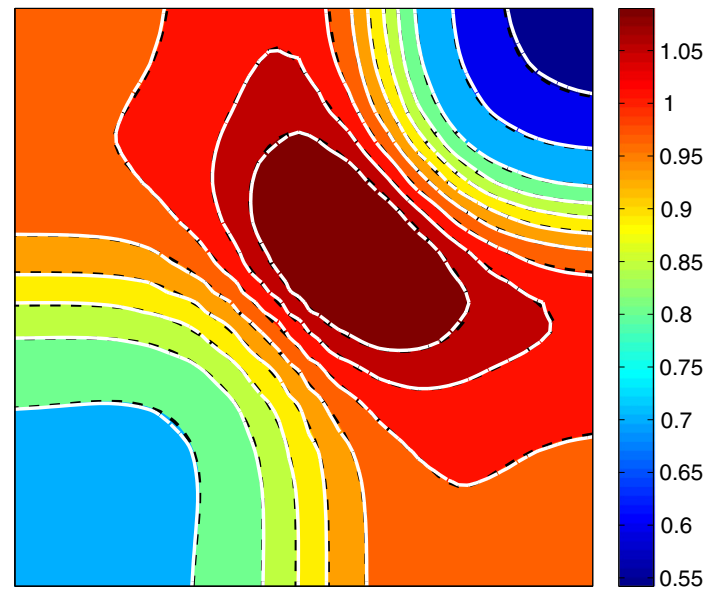

(c)

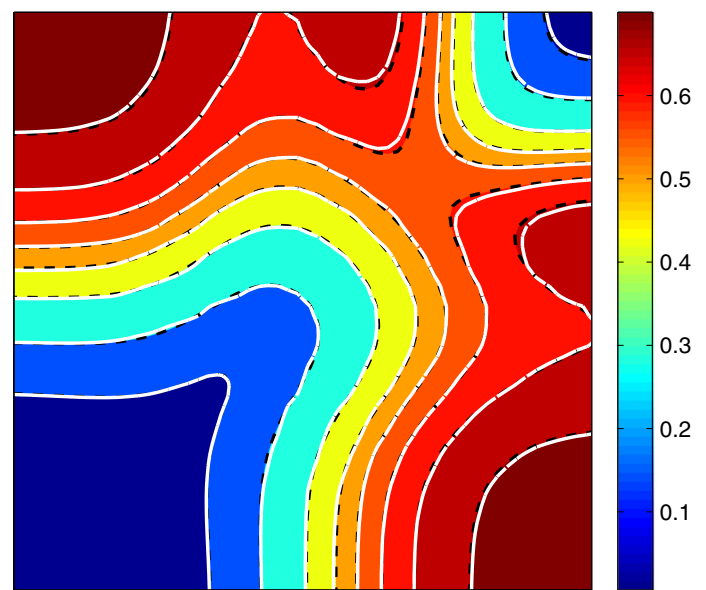

(b)

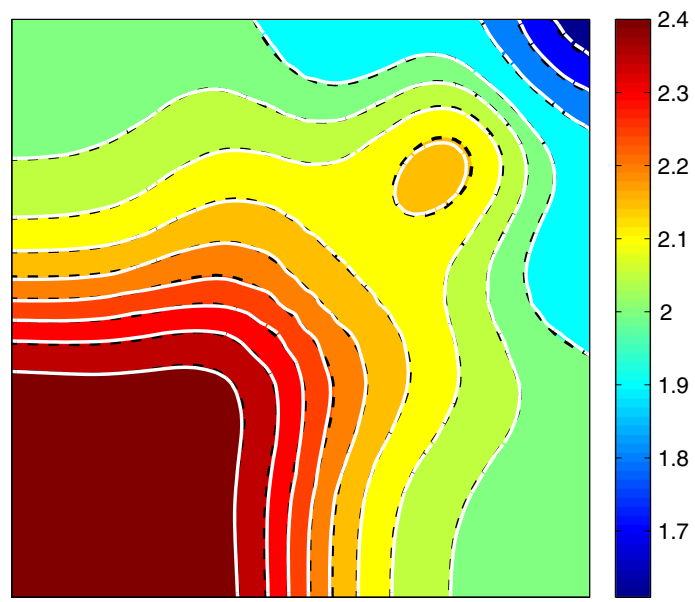

(d)

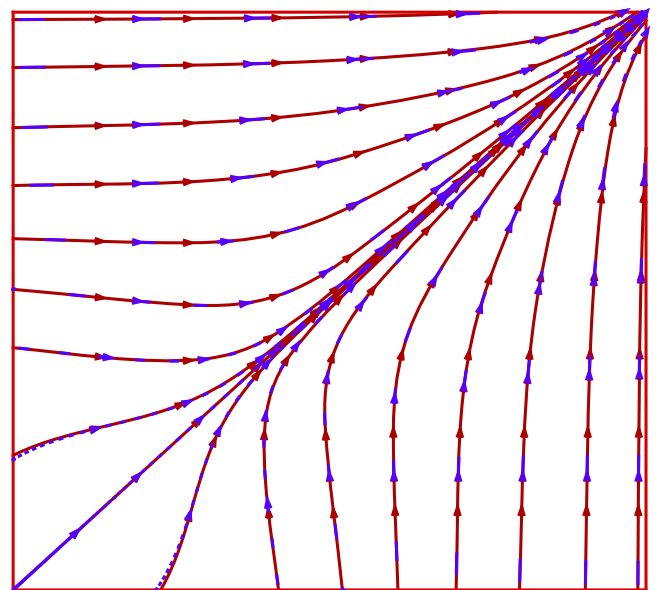

FIG. 9. (Color online) Contours of the density (a), temperature (b), velocity magnitude (c), and velocity streamlines (d) of the 2D Riemann problem at $\mu_{0}=10$. In (a)-(c), the background and dashed lines are from the collisionless Boltzmann equation, and the solid lines are the DUGKS results. In (d), the dashed lines are the solutions of collisionless Boltzmann equation, and the solid lines are the DUGKS results.

configuration is in excellent agreement with the solution of Euler equations by different numerical methods (e.g., [39,40]). It is noted that the temperature configuration for this problem was rarely presented in previous studies, and here we show it just for a reference. But we can observe from Fig. 8 that the temperature structure is consistent with the density structure.

We now test the DUGKS for the problem in the freemolecular regime by choosing $\mu_{0}=10$. In this case the flow is highly nonequilibrium, although the flow field is smooth. In order to capture the nonequilibrium effects, the particle velocity space is discretized with a $201 \times 201$ mesh points in $[-15,15] \times[-15,15]$ based on the Cotes quadrature. Furthermore, a uniform mesh with $60 \times 60$ cells is used in the physical space, which is sufficient to obtain well-resolved solutions. In Fig. 9 the density, temperature, velocity magnitude $\left[\left(u_{x}^{2}+u_{y}^{2}\right)^{1 / 2}\right]$, and streamlines are shown at $t=0.15$. For comparison, the results from the solution of collisionless Boltzmann equation are also presented (see Appendix B). It can be seen that the flow patterns predicted by the DUGKS are in excellent agreement with the analytical solutions of the collisionless Boltzmann equation. The agreement with available data in both continuum and free-molecular regimes suggests that the DUGKS has a nice dynamic adaptive property for multiregime flows, which is desirable for multiscale flow simulations.

\section{SUMMARY}

The capturing of multiscale flow physics of different regimes uniformly in a numerical scheme is difficult. In this paper, a finite-volume discrete unified gas kinetic scheme is developed for multiregime flows. With the implementation of coupled transport and collision along the characteristic line of the kinetic equation in the determination of the gas distribution function and flux at a cell interface, the DUGKS can simulate flows in different regimes accurately. Particularly, the time step and cell size in DUGKS are not limited by the particle collision time and mean free path of gas molecules. With the variation of the ratio of the time step to the local particle collision time, a continuum spectrum of flow physics from the rarefied to the continuum flow regimes can be fully recovered. What is important for the DUGKS is that it provides a reliable tool for flow simulation with the coexistence of both continuum and rarefied flow regimes. The DUGKS has second-order 
accuracy, multidimensional nature, and asymptotic preserving properties, and the scheme is validated by test problems ranging from continuum to free-molecular flows. The analysis and numerical results suggest that the DUGKS is a valuable method for the multiscale flow physics computation. It is interesting to note that the DUGKS and UGKS methods present similar numerical results, and the DUGKS is about $30 \%$ faster than UGKS with the same computational mesh and discrete velocity set.

As a continuation of research in this direction, the DUGKS can be further improved in several aspects. First, it is well known that the Shakhov model with a velocity-independent relaxation time is inadequate for highly rarefied supersonic nonequilibrium flow simulation, especially for the flow with significant temperature variation, such as the temperature and heat flux profiles inside the strong shock structure. A velocitydependent relaxation model or the full Boltzmann collision operator may be employed to improve the prediction of the DUGKS for these cases [42], which is particularly useful for high-Mach-number flows. Even though it is easy to incorporate a generalized relaxation model to the present DUGKS, it will become difficult to directly include the full Boltzmann collision term in the DUGKS due to the combined update of distribution function and collision effect. Other strategies need to be used to remove such a limitation. For instance, similar to the UGKS method, we may update the original distributions and the conservative variables at both cell centers and cell interfaces simultaneously to avoid the introductions of $\tilde{f}$ and $\bar{f}$, and then extend the DUGKS to include the full Boltzmann collision operator which has been used in the improved UGKS [23]. The second possible improvement lies in the further improvement of accuracy. The present DUGKS has second-order accuracy in both space and time, which is the same as the UGKS method. For example, the time accuracy can be improved by employing a high-order Runge-Kutta method for time evolution, and the spatial accuracy can be enhanced with the high-order initial reconstruction for the distribution function around a cell interface. In this case, the real gas distribution function instead of the combined function of the current method should be employed. This may be achieved by following the strategy used in the UGKS [14]. Another possible improvement is on the discrete particle velocity set. Like other deterministic numerical methods for kinetic equations, a set of discrete velocities should be employed in the present DUGKS, which may be very large for high-speed and/or highly nonequilibrium flows. This will lead to a large amount of computer memory and high computational cost. The use of adaptive velocity techniques can improve the computational efficiency greatly $[43,44]$. All these aspects for the further development of DUGKS will be considered in our future work.

Finally, we would like to point out that the present DUGKS method can be expended to other multiscale transport problems, such as multiple species and multiphase systems [29]. The kinetic collision operators for these systems are more complicated, and the interactions among different species or phases have to be taken into account. But based on the relaxation kinetic model, a DUGKS can be constructed following the similar procedure presented in the current work.

\section{ACKNOWLEDGMENTS}

Z.L.G. acknowledges support from the National Natural Science Foundation of China (No. 51125024), and part of the work was carried out during his visit to the Hong Kong University of Science and Technology. K.X. was supported by the Hong Kong Research Grant Council (Grants No. 621011 and No. 620813).

\section{APPENDIX A: SOLUTION OF COLLISIONLESS BOLTZMANN EQUATION FOR THE 1D SHOCK TUBE PROBLEM}

For the shock tube problem, the solution of the collisionless Boltzmann equation is

$$
f(x, \xi, t)= \begin{cases}f^{e q}\left(\boldsymbol{W}_{L}\right), & \xi \geqslant x / t, \\ f^{e q}\left(\boldsymbol{W}_{R}\right), & \xi<x / t .\end{cases}
$$

By taking velocity moments of $f(x, \xi, t)$, we can obtain the conservative variables,

$$
\begin{aligned}
\rho(x, t)= & \frac{\rho_{1}}{2} \operatorname{erfc}\left(-\tilde{u}_{1}\right)+\frac{\rho_{2}}{2} \operatorname{erfc}\left(\tilde{u}_{2}\right), \\
\rho u(x, t)= & \frac{\rho_{1}}{2}\left[\left(2 R T_{1} / \pi\right)^{1 / 2} \exp \left(-\tilde{u}_{1}^{2}\right)+u_{1} \operatorname{erfc}\left(-\tilde{u}_{1}\right)\right]+\frac{\rho_{2}}{2}\left[\left(2 R T_{2} / \pi\right)^{1 / 2} \exp \left(-\tilde{u}_{2}^{2}\right)-u_{2} \operatorname{erfc}\left(\tilde{u}_{2}\right)\right], \\
\rho E(x, t)= & \frac{\rho_{1}}{4}\left\{\left[u_{1}^{2}+(K+3) R T_{1}\right] \operatorname{erfc}\left(-\tilde{u}_{1}\right)+\left(u_{1}+x / t\right)\left(2 R T_{2} / \pi\right)^{1 / 2} \exp \left(-\tilde{u}_{2}^{2}\right)\right\} \\
& +\frac{\rho_{2}}{4}\left\{\left[u_{2}^{2}+(K+3) R T_{2}\right] \operatorname{erfc}\left(\tilde{u}_{2}\right)-\left(u_{2}+x / t\right)\left(2 R T_{2} / \pi\right)^{1 / 2} \exp \left(-\tilde{u}_{2}^{2}\right)\right\}
\end{aligned}
$$

where $\tilde{u}_{i}=\left(u_{i}-x / t\right) / \sqrt{\left(2 R T_{i}\right)}$, and erfc is the complementary error function defined by

$$
\operatorname{erfc}(z)=\frac{2}{\sqrt{\pi}} \int_{z}^{\infty} e^{-t^{2}} d t
$$




\section{APPENDIX B: SOLUTION OF COLLISIONLESS BOLTZMANN EQUATION FOR THE 2D RIEMANN PROBLEM}

For the 2D Riemann problem, the solution of the collisionless Boltzmann equation is

$$
f\left(x, y, \xi_{x}, \xi_{y}, \boldsymbol{\eta}, t\right)=f^{e q}\left(x-\xi_{x} t, y-\xi_{y} t, \xi_{x}, \xi_{y}, \eta, 0\right) .
$$

Then the conservative variables can be obtained by taking the velocity moments of $f$,

$$
\begin{aligned}
\rho(x, y, t)= & \frac{\rho_{1}}{4} \operatorname{erfc}\left(\tilde{u}_{1}\right) \operatorname{erfc}\left(\tilde{v}_{1}\right)+\frac{\rho_{2}}{4} \operatorname{erfc}\left(-\tilde{u}_{2}\right) \operatorname{erfc}\left(\tilde{v}_{2}\right)+\frac{\rho_{3}}{4} \operatorname{erfc}\left(-\tilde{u}_{3}\right) \operatorname{erfc}\left(-\tilde{v}_{3}\right)+\frac{\rho_{4}}{4} \operatorname{erfc}\left(\tilde{u}_{4}\right) \operatorname{erfc}\left(-\tilde{v}_{4}\right), \\
\rho u(x, y, t)= & \frac{\rho_{1}}{4}\left[-\left(2 R T_{1} / \pi\right)^{1 / 2} e^{-\tilde{u}_{1}^{2}}+u_{1} \operatorname{erfc}\left(\tilde{u}_{1}\right)\right] \operatorname{erfc}\left(\tilde{v}_{1}\right)+\frac{\rho_{2}}{4}\left[\left(2 R T_{2} / \pi\right)^{1 / 2} e^{-\tilde{u}_{2}^{2}}+u_{2} \operatorname{erfc}\left(-\tilde{u}_{2}\right)\right] \operatorname{erfc}\left(\tilde{v}_{2}\right) \\
& +\frac{\rho_{3}}{4}\left[\left(2 R T_{3} / \pi\right)^{1 / 2} e^{-\tilde{u}_{3}^{2}}+u_{3} \operatorname{erfc}\left(-\tilde{u}_{3}\right)\right] \operatorname{erfc}\left(-\tilde{v}_{3}\right)+\frac{\rho_{4}}{4}\left[-\left(2 R T_{4} / \pi\right)^{1 / 2} e^{-\tilde{u}_{4}^{2}}+u_{4} \operatorname{erfc}\left(\tilde{u}_{4}\right)\right] \operatorname{erfc}\left(-\tilde{v}_{4}\right), \\
\rho v(x, y, t)= & \frac{\rho_{1}}{4}\left[-\left(2 R T_{1} / \pi\right)^{1 / 2} e^{-\tilde{v}_{1}^{2}}+v_{1} \operatorname{erfc}\left(\tilde{v}_{1}\right)\right] \operatorname{erfc}\left(\tilde{u}_{1}\right)+\frac{\rho_{2}}{4}\left[-\left(2 R T_{2} / \pi\right)^{1 / 2} e^{-\tilde{v}_{2}^{2}}+v_{2} \operatorname{erfc}\left(-\tilde{v}_{2}\right)\right] \operatorname{erfc}\left(-\tilde{u}_{2}\right) \\
& +\frac{\rho_{3}}{4}\left[\left(2 R T_{3} / \pi\right)^{1 / 2} e^{-\tilde{v}_{3}^{2}}+v_{3} \operatorname{erfc}\left(-\tilde{v}_{3}\right)\right] \operatorname{erfc}\left(-\tilde{u}_{3}\right)+\frac{\rho_{4}}{4}\left[-\left(2 R T_{4} / \pi\right)^{1 / 2} e^{-\tilde{v}_{4}^{2}}+v_{4} \operatorname{erfc}\left(\tilde{v}_{4}\right)\right] \operatorname{erfc}\left(\tilde{u}_{4}\right),
\end{aligned}
$$

and

$$
\rho E(x, y, t)=\frac{1}{8}\left(\rho_{1} J_{1}+\rho_{2} J_{2}+\rho_{3} J_{3}+\rho_{4} J_{4}\right),
$$

with

$$
\begin{aligned}
J_{1}(x, y, t)= & -\left[\left(y / t+v_{1}\right) e^{-\tilde{v}_{1}^{2}} \operatorname{erfc}\left(\tilde{u}_{1}\right)+\left(x / t+u_{1}\right) e^{-\tilde{u}_{1}^{2}} \operatorname{erfc}\left(\tilde{v}_{1}\right)\right]\left(2 R T_{1} / \pi\right)^{1 / 2} \\
& +\left[(K+2) R T_{1}+u_{1}^{2}+v_{1}^{2}\right] \operatorname{erfc}\left(\tilde{u}_{1}\right) \operatorname{erfc}\left(\tilde{v}_{1}\right), \\
J_{2}(x, y, t)= & -\left[\left(y / t+v_{2}\right) e^{-\tilde{v}_{2}^{2}} \operatorname{erfc}\left(-\tilde{u}_{2}\right)-\left(x / t+u_{2}\right) e^{-\tilde{u}_{2}^{2}} \operatorname{erfc}\left(\tilde{v}_{2}\right)\right]\left(2 R T_{2} / \pi\right)^{1 / 2} \\
& +\left[(K+2) R T_{2}+u_{2}^{2}+v_{2}^{2}\right] \operatorname{erfc}\left(-\tilde{u}_{2}\right) \operatorname{erfc}\left(\tilde{v}_{2}\right), \\
J_{3}(x, y, t)= & {\left[\left(y / t+v_{2}\right) e^{-\tilde{v}_{3}^{2}} \operatorname{erfc}\left(-\tilde{u}_{3}\right)+\left(x / t+u_{3}\right) e^{-\tilde{u}_{3}^{2}} \operatorname{erfc}\left(-\tilde{v}_{3}\right)\right]\left(2 R T_{3} / \pi\right)^{1 / 2} } \\
& +\left[(K+2) R T_{3}+u_{3}^{2}+v_{3}^{2}\right] \operatorname{erfc}\left(-\tilde{u}_{3}\right) \operatorname{erfc}\left(-\tilde{v}_{3}\right), \\
J_{4}(x, y, t)= & {\left[\left(y / t+v_{4}\right) e^{-\tilde{v}_{4}^{2}} \operatorname{erfc}\left(\tilde{u}_{4}\right)-\left(x / t+u_{4}\right) e^{-\tilde{u}_{4}^{2}} \operatorname{erfc}\left(-\tilde{v}_{4}\right)\right]\left(2 R T_{4} / \pi\right)^{1 / 2} } \\
& +\left[(K+2) R T_{4}+u_{4}^{2}+v_{4}^{2}\right] \operatorname{erfc}\left(\tilde{u}_{4}\right) \operatorname{erfc}\left(-\tilde{v}_{4}\right),
\end{aligned}
$$

where $\tilde{\boldsymbol{u}}_{i}=\left(\boldsymbol{u}_{i}-\boldsymbol{x} / t\right) / \sqrt{2 R T_{i}}$.

[1] G. A. Radtke, J.-P. M. Péraud, and N. G. Hadjiconstantinou, Philos. Trans. R. Soc., A 371, 20120182 (2012).

[2] S. T. O'Connell and P. A. Thompson, Phys. Rev. E 52, R5792 (1995).

[3] T. Werder, J. H. Walther, and P. Koumoutsakos, J. Comput. Phys. 205, 373 (2005).

[4] W. N. E, B. Engquist, and Z. Huang, Phys. Rev. B 67, 092101 (2003).

[5] M. K. Borg, D. A. Lockerby, and J. M. Reese, J. Comput. Phys. 255, 149 (2013).

[6] H. A. Carlson, R. Roveda, I. D. Boyd, and G. V. Candler, AIAA Paper 2004-1180 (2004).

[7] J. Y. Yang and J. C. Huang, J. Comput. Phys. 120, 323 (1995).

[8] Z. H. Li and H. X. Zhang, J. Comput. Phys. 193, 708 (2004).

[9] A. N. Kudryavtsev and A. A. Shershnev, J. Sci. Comput. 57, 42 (2013).

[10] S. Pieraccini and G. Puppo, J. Sci. Comput. 32, 1 (2007).

[11] M. Bennoune, M. Lemo, and L. Mieussens, J. Comput. Phys. 227, 3781 (2008).

[12] F. Filbet and S. Jin, J. Comput. Phys. 229, 7625 (2010).
[13] G. Dimarco and L. Pareschi, Numer. Anal. 51, 1064 (2013).

[14] K. Xu and J.-C. Huang, J. Comput. Phys. 229, 7747 (2010).

[15] J. C. Huang, K. Xu, and P. B. Yu, Commun. Comput. Phys. 12, 662 (2012).

[16] P. L. Bhatnagar, E. P. Gross, and M. Krook, Phys. Rev. 94, 511 (1954).

[17] Z. L. Guo, K. Xu, and R. J. Wang, Phys. Rev. E 88, 033305 (2013).

[18] E. M. Shakhov, Fluid Dyn. 3, 95 (1968).

[19] L. H. Holway, Phys. Fluids 9, 1658 (1966).

[20] B. van Leer, J. Comput. Phys. 23, 276 (1977).

[21] L. Mieussens, J. Comput. Phys. 253, 138 (2013).

[22] K. Xu, J. Comput. Phys. 171, 289 (2001).

[23] C. Liu, K. Xu, Q. H. Sun, and Q. D. Cai, arXiv:1405.4479[math.NA].

[24] G. A. Bird, Molecular Gas Dynamics and the Direct Simulation of Gas Flows (Clarendon Press, Oxford, 1994).

[25] J. Fan and C. Shen, J. Comput. Phys. 167, 393 (2001).

[26] L. L. Baker and N. G. Hadjiconstantinou, Phys. Fluids 17, 051703 (2005). 
[27] Z. L. Guo and C. Shu, Lattice Boltzmann Method and its Applications in Engineering (World Scientific Publishing, Singapore, 2013).

[28] P. Wang, L. H. Zhu, Z. L. Guo, and K. Xu, Commun. Comput. Phys. 17, 657 (2015).

[29] S. Harris, An Introduction to the Theory of the Boltzmann Equation (Dover Publications, New York, 2004).

[30] T. Ohwada, Phys. Fluids A 5, 217 (1993).

[31] K. Xu and J.-C. Huang, IMA J. Appl. Math. 76, 698 (2011).

[32] S. Liu and C. W. Zhong, Phys. Rev. E 89, 033306 (2014).

[33] G. A. Bird, Phys. Fluids 13, 1172 (1970).

[34] G. A. Sod, J. Comput. Phys. 27, 1 (1978).

[35] C. W. Schulz-Rinne, SIAM J. Math. Anal. 24, 76 (1993).
[36] C. W. Schulz-Rinne, J. P. Collins, and H. M. Glaz, SIAM J. Sci. Comput. 14, 1394 (1993).

[37] T. Zhang and Y. Zheng, SIAM J. Math. Anal. 21, 593 (1990).

[38] T. Chang, G.-Q. Chen, and S. Yang, Disc. Cont. Dyn. Syst. 1, 555 (1995); 6, 419 (2000).

[39] P. Lax and X.-D. Liu, SIAM J. Sci. Comput. 19, 319 (1998).

[40] A. Kurganov and E. Tadmor, Numer. Meth. Part. Differ. Equ. 18, 584 (2002).

[41] B. Shizgal, J. Comput. Phys. 41, 309 (1981).

[42] L. Wu, J. M. Reese, and Y. H. Zhang, J. Fluid Mech. 746, 53 (2014).

[43] S. Z. Chen, K. Xu, C. B. Li, and Q. D. Cai, J. Comput. Phys. 231, 6643 (2012).

[44] S. Brull and L. Mieussens, J. Comput. Phys. 266, 22 (2014). 